\title{
Upscaling and reversibility of Taylor dispersion in heterogeneous porous media
}

\author{
C. W. J. Berentsen, ${ }^{*}$ M. L. Verlaan, ${ }^{\dagger}$ and C. P. J. W. van Kruijsdijk ${ }^{+}$ \\ Department of Geotechnology, Delft University of Technology, Mijnbouwstraat 120, Delft, The Netherlands
}

(Received 9 July 2004; revised manuscript received 17 November 2004; published 29 April 2005)

\begin{abstract}
Tracer flow in stratified porous media is dominated by the interaction between convective transport and transverse diffusive mixing. By averaging the tracer concentration in the transverse direction, a onedimensional non-Fickian dispersion model is derived. The model accounts for the relaxation process that reduces the convective transport to dispersive mixing. This process is (short-) time correlated and partially reversible upon reversal of flow direction. For multiscale velocity fields, the relaxation is a multiscale process. To date only single scale processes have been successfully upscaled. Our procedure extends this to multiscale processes, using scale separation. The model parameters can be calculated a priori based on the velocity profile. For periodic flow reversal, the results are essentially the same. Despite the non-Fickian behavior during a cycle, the net contribution of each cycle to the spreading relaxes to a Fickian process in a similar way as for unidirectional flow. The cycle time averaged dispersion coefficient is a monotonically increasing function of the reversal time. It asymptotically converges towards the effective dispersion coefficient in the absence of flow reversal.
\end{abstract}

DOI: 10.1103/PhysRevE.71.046308 PACS number(s): 47.55.Mh, 47.55.Hd, 05.60.-k, 05.70.Ln

\section{INTRODUCTION}

Considering convective dispersion, there are a number of situations where the physical mechanisms are not well described by the classical convection-dispersion equation (CCDE).

(i) In natural rocks, often several scales of heterogeneities (e.g., layers) are encountered which exhibit a different dispersion behavior from those that can be obtained from the CCDE [1,2].

(ii) The interaction between convection and molecular diffusion can be significant [3-8].

(iii) When the flow direction is reversed, convective spreading will also reverse. The CCDE describes the dispersion as an irreversible mixing mechanism $[9,10]$.

Numerous researchers modified the CCDE to fit their problem. The modifications range from extending the model for the macroscopic dispersion coefficient $[1,11,12]$ to adding extra terms to the CCDE $[2,13,14]$. However, none of the extended models is capable of handling all three situations outlined above. This paper describes a method for upscaling of dispersion in arbitrary layered porous media to obtain a macroscopically averaged model which incorporates the above-mentioned physical mechanisms. It extends the approach taken by Camacho $[15,16]$. The emphasis of this paper is on the validity of the upscaled model and the relation between the parameters of the model and the physics of the process.

\footnotetext{
*Present address: Department of Earth Sciences, Utrecht University. Electronic address: berentsen@ geo.uu.nl

${ }^{\dagger}$ Present address: Horizon Energy Partners. Electronic address: marco.verlaan@horizon-ep.com

ثElectronic address: c.p.j.w.vankruijsdijk@citg.tudelft.nl
}

\section{PHYSICS-DISPERSION IN A UNIDIRECTIONAL FLOW FIELD}

Consider a macroscopically stationary unidirectional velocity field $\mathrm{v}(\mathrm{y})$, which corresponds to flow through an infinite two-dimensional porous media bounded in the transverse or $y$ direction, see Fig. 1. At $t=0$, we inject a finite mass $\left(\mathrm{M}_{0}\right)$ of ideal [24] tracer (non)uniformly distributed over the height $(\mathrm{y})$ at longitudinal position $\mathrm{x}_{0}$. In addition to convective transport, the tracer particles are subject to a microscale mixing mechanism, which we will limit in this paper to isotropic molecular diffusion [25]. The evolution of the (tracer) concentration can be described, at the macroscale, by the two-dimensional unidirectional convectiondiffusion equation (2D uCDifE),

$$
\frac{\partial c(x, y, t)}{\partial t}+v(y) \frac{\partial c(x, y, t)}{\partial x}=D_{m o l} \Delta c(x, y, t) .
$$

Our objective is to describe the evolution of the transverse averaged concentration $c_{0}(x, t)=(1 / d) \int_{0}^{d} c(x, y, t) d y$.

\section{A. Convective displacement}

First consider convective transport only. Each tracer particle moves with a constant velocity in the $\mathrm{x}$ direction along its initial streamline. Hence, the mean position of all tracer particles moves linearly in time with a constant averaged particle velocity $\left(u_{0}\right)$. This velocity may differ from the mean fluid velocity $\left(v_{0}\right)$ if the particles are nonuniformly distributed in the transverse direction. Similarly, the spreading of the particles in the longitudinal direction, expressed in terms of the variance, increases with time squared and is proportional to the variance of the tracer velocity determined by those streamlines occupied by tracer particles. 


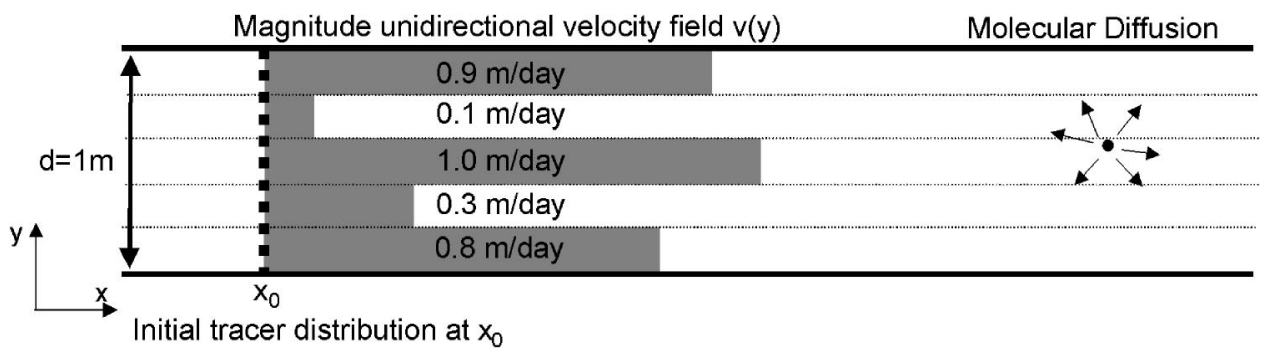

FIG. 1. Layered porous media in the domain $\Omega=(-\infty,+\infty) \times[0, d]$ in which a longitudinal velocity field $\mathrm{v}(\mathrm{y})$ is present. Tracer particles are initially released in this field at longitudinal position $x_{0}$ uniformly or nonuniformly distributed in the transverse direction. Tracer particles are subject to isotropic molecular diffusion.

\section{B. Relaxation from convective towards uncorrelated-Fickian behavior}

In addition to convection, consider mixing by molecular diffusion. The longitudinal component of diffusion smoothes the sharp front propagated by convection. More importantly, in the transverse direction diffusion moves particles away from their initial streamlines and the particle velocity will change. Over time, each tracer particle will sample each vertical position equally frequently, and the relation between a particle and its initial streamline is lost. Consequently, diffusion smoothes concentration differences in the transverse direction, and as a result reduces the longitudinal spreading caused by the velocity profile. In the long-time limit, a dynamic equilibrium between the convective and diffusive spreading mechanisms is established, which exhibits Fickian behavior. Figure 2 visualizes this process for the velocity field shown in Fig. 1. The relaxation time $\tau$ is the characteristic time for the transition process [26] also called relaxation. This time is a function of the value of the molecular diffusion coefficient and the correlation of the longitudinal velocity in the transverse direction.

\section{The effect of the initial transverse particle distribution}

If the particle distribution is uniform over the height, local redistribution of the particles by molecular diffusion does not affect the particle distribution over the streamlines. The mean particle velocity equals the mean fluid velocity at all times. If the initial particle distribution is not uniform, transverse diffusion will establish a uniform distribution over the height in time and the mean particle velocity evolves from its initial value $\mathrm{u}_{0}$ to the mean fluid velocity $\mathrm{v}_{0}$.

\section{SPECTRAL EQUIVALENT}

Similar to Camacho [16], the starting point for the derivation of an evolution equation for the height averaged concentration $\left[c_{0}(x, t)\right]$ is the spectral equivalent of the $2 \mathrm{D}$ uCDifE. We replace the concentration and velocity (1) by cosine Fourier series, multiply the result by $\cos (n \pi y / d)$, and integrate it over $\mathrm{y}$. For $\mathrm{n}=0$, we obtain the evolution equation of $c_{0}(x, t)$,

$$
\frac{\partial c_{0}}{\partial t}+v_{0} \frac{\partial c_{0}}{\partial x}-D_{m o l} \frac{\partial^{2} c_{0}}{\partial x^{2}}=-\frac{\partial J_{T}}{\partial x}
$$

where, following Camacho [16], the single valued dispersive Taylor flux $J_{T}$ is defined as

$$
J_{T}(x, t)=\frac{1}{2} \sum_{n=1}^{\infty} v_{n} c_{n}(x, t) .
$$

It originates from the convective term in Eq. (1) and accounts for the contribution of the higher-order modes to $c_{0}(x, t)$. The evolution of the higher-order modes $(n>0)$ is given by

$$
\frac{\partial c_{n}}{\partial t}+\frac{c_{n}}{\tau_{n}}+v_{0} \frac{\partial c_{n}}{\partial x}+\sum_{m=1}^{\infty} \frac{\gamma_{m n}}{v_{n}} v_{m} \frac{\partial c_{m}}{\partial x}-D_{m o l} \frac{\partial^{2} c_{n}}{\partial x^{2}}=-v_{n} \frac{\partial c_{0}}{\partial x},
$$

where the modal relaxation time $\tau_{n}$ and modal interaction coefficient $\gamma_{m n}$ are defined as

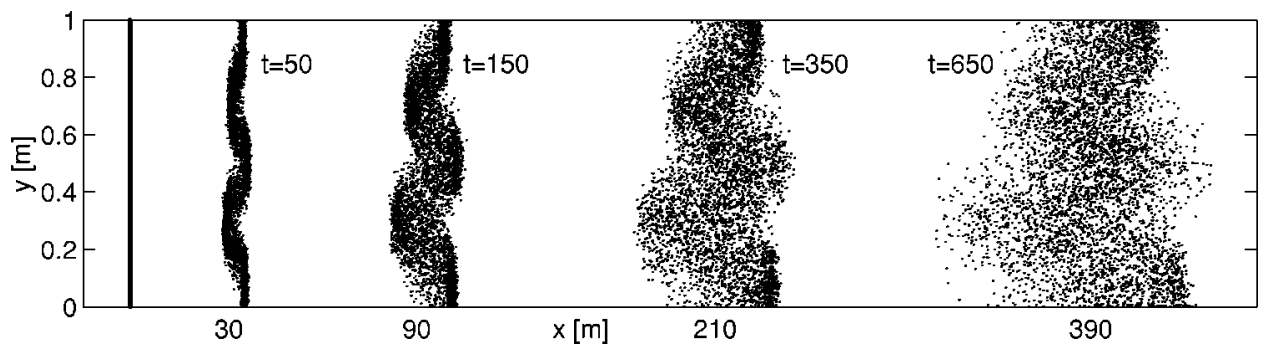

FIG. 2. Typical evolution of particle clouds in the $(x, y)$ space when the particles are initially uniformly distributed over the transverse direction. Clouds at increasing position $(\mathrm{x})$ represent clouds for increasing time. Times $(\mathrm{t})$ are in days. The molecular diffusion is $\mathrm{D}_{m o l}$ $=1.25 \times 10^{-4} \mathrm{~m}^{2} / \mathrm{d}$. 


$$
\tau_{n}=\frac{d^{2}}{(n \pi)^{2} D_{m o l}}
$$

and

$$
\gamma_{m n}= \begin{cases}\frac{1}{2} \frac{v_{n}}{v_{m}}\left[v_{m+n}\right], & m=n \\ \frac{1}{2} \frac{v_{n}}{v_{m}}\left[v_{m+n}+v_{|m-n|}\right], & m \neq n .\end{cases}
$$

\section{MOMENT ANALYSIS FOR UNIDIRECTIONAL FLOW}

The spectral representation also serves as a starting point for the derivation of the spatial moments belonging to the $2 \mathrm{D}$ uCDifE. As the 2D model is generally too involved to allow direct analytical solution, moment analysis allows us to quantify the accuracy of an upscaled model. We briefly discuss the behavior of the zeroth, first, and second spatial moments belonging to the $2 \mathrm{D}$ uCDifE. For a full analysis, see $[5,17,18]$.

\section{A. General definition of the spatial moments}

Consider the release of a tracer with finite mass $\mathrm{M}_{0}$ in the spatial domain $x \in(-\infty,+\infty)$. The $k$ th spatial moment of the $n$th concentration mode is defined as

$$
E_{c_{n}, x, k}=\int_{-\infty}^{+\infty} x^{k} c_{n}(x) d x .
$$

The zeroth moments or "mass" in the $n$th concentration mode are denoted by $M_{c_{n}} . M_{c_{n}, x, k}$ is the tracer mass normalized $k$ th moment of the $n$th concentration mode, $\left(E_{c_{n}, x, k} / M_{0}\right)$. $M_{c_{n}, x, k}^{c}$, for $k>1$, is the centerd normalized $k$ th moment $\left[M_{c_{n},\left(x-M_{c, x, 1}\right), k}\right]$. For simplicity, the mean particle position $\left(M_{c_{0}, x, 1}\right)$ is written as $\mu_{c, x}$ and the particle variance $\left(M_{c_{0}, x, 2}^{c}\right)$ as $\sigma_{c, x}^{2}$.

\section{B. The evolution of the spatial moments of the spectral equivalent}

To obtain the moment equations belonging to the $2 \mathrm{D}$ uCDifE, we multiply Eqs. (2) and (4) of the spectral equivalent with $\mathrm{x}^{k}$ and average the result over $\mathrm{x}$. Assuming that each concentration mode and all its spatial derivatives converge exponentially to zero for $\mathrm{x} \rightarrow \pm \infty$, the evolution equation for the $k$ th normalized moment belonging to $c_{0}(x, t)$ can be written as

$$
\begin{aligned}
& \frac{\partial M_{c_{0}, x, k}}{\partial t}-k v_{0} M_{c_{0}, x, k-1}-\left(k^{2}-k\right) D_{m o l} M_{c_{0}, x, k-2} \\
& \quad=\frac{k}{2} \sum_{n=1}^{\infty} v_{n} \frac{E_{c_{n}, x, k-1}}{M_{c_{0}}} .
\end{aligned}
$$

The evolution of the $k$ th non-normalized moment of a higher concentration mode $(n>0)$ reads

$$
\begin{aligned}
{\left[\tau_{n}^{-1}+\frac{\partial}{\partial t}\right] E_{c_{n}, x, k}=} & k v_{n} M_{c_{0}} M_{c_{0}, x, k-1}+k\left[2 v_{0}+v_{2 n}\right] E_{c_{n}, x, k-1} \\
& +\left(k^{2}-k\right) D_{m o l} E_{c_{n}, x, k-2} \\
& +\frac{k}{2} \sum_{m=1 \neq n}^{\infty}\left[v_{m+n}+v_{|m-n|}\right] E_{c_{n}, x, k-1}
\end{aligned}
$$

\section{The transverse particle distribution}

If the tracer is initially nonuniformly distributed over the height, the initial zeroth moment ("mass") of a higher concentration mode $c_{n}(n>0)$ may be nonzero,

$$
M_{c_{n}}^{\text {init }}=2 \int_{-\infty}^{+\infty} \int_{0}^{d} c(x, y, 0) \cos \left(\frac{n \pi y}{d}\right) d y d x .
$$

Solving Eq. (9) for $\mathrm{k}=0$ with respect to initial condition (10) gives the evolution of the zeroth moment of the higherorder modes $(n>0)$,

$$
M_{c_{n}}(t)=M_{c_{n}}^{i n i t} e^{-t / \tau_{n}} .
$$

Equation (11) shows that the zeroth moment of $c_{n}$ relaxes to zero at a speed which only depends on its own relaxation time $\tau_{n}$. Its relaxation is not affected by other concentration modes.

\section{The mean particle position and velocity}

If the particles are distributed nonuniformly in the transverse direction, the mean particle velocity $[\mathrm{u}(\mathrm{t})]$ shows a relaxation from the initial mean particle velocity $\left[\mathrm{u}_{0}=\mathrm{u}(0)\right]$, determined by the initially occupied streamlines, towards the mean fluid velocity $\left(\mathrm{v}_{0}\right)$,

$$
u(t)=\frac{\int_{-\infty}^{+\infty} \int_{0}^{d} v c d y d x}{\int_{-\infty}^{+\infty} \int_{0}^{d} c d y d x}=v_{0}+\frac{1}{2} \sum_{n=1}^{\infty} v_{n} \frac{M_{c_{n}}^{\text {init }}}{M_{0}} e^{-t / \tau_{n}}
$$

Convergence from $\mathrm{u}_{0}$ to $\mathrm{v}_{0}$ is faster for increasing magnitude of the molecular diffusion $\mathrm{D}_{m o l}$, as shown in Fig. 3.

The exact mean position of the particles is obtained from Eq. (8) with $\mathrm{k}=1$ or by integration of the mean particle velocity (12) over time,

$$
\mu_{c, x}=\left[x_{0}+v_{0} t\right]+\frac{1}{2} \sum_{n=1}^{\infty} \tau_{n} v_{n} \frac{M_{c_{n}}^{\text {init }}}{M_{0}}\left[1-e^{-t / \tau_{n}}\right] .
$$

In addition to a contribution present for initial uniform particle distributions $\left(x_{0}+v_{0} t\right)$, a relaxation term expresses the contribution due to the redistribution of the particles over the streamlines. After relaxation, the mean moves with the mean fluid velocity as in the uniform case, but shows a deviation $\Delta \mu_{\text {relax }}$ resulting from the relaxation of the mean particle velocity, 


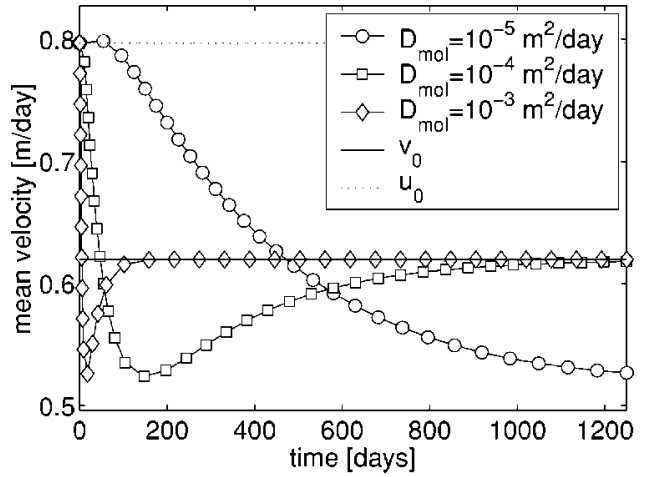

FIG. 3. Typical example of the relaxation of the mean particle velocity towards the mean fluid velocity $\mathrm{v}_{0}$ for three values of the molecular diffusion.

$$
\mu_{c, x, \text { disp }}=x_{0}+v_{0} t+\sum_{n=1}^{\infty} \frac{\tau_{n} v_{n}}{2} \frac{M_{c_{n}}^{\text {init }}}{M_{0}}=x_{0}+v_{0} t+\Delta \mu_{\text {relax }} \text {. }
$$

\section{E. The variance of the averaged concentration}

The spatial variance is given by (for derivation, see Appendix A 2)

$$
\begin{aligned}
\sigma_{c, x}^{2}(t)= & \left(2 D_{m o l} t\right)+\left(\sum_{n=1}^{\infty} v_{n}^{2} \tau_{n}\left(t+\tau_{n}\left[e^{-t / \tau_{n}}-1\right]\right)\right) \\
& -\left(\frac{1}{2} \sum_{n=1}^{\infty} \frac{M_{c_{n}}^{i n i t}}{M_{0}} v_{n} \tau_{n}\left[1-e^{\left.-t / \tau_{n}\right]}\right)^{2}\right. \\
& +\left(\frac{1}{2} \sum_{n=1}^{\infty} \frac{M_{c_{n}}^{\text {init }}}{M_{0}} v_{n} v_{2 n}\left(\tau_{n}^{2}-\left[\tau_{n}^{2}+\tau_{n} t\right] e^{-t / \tau_{n}}\right)\right. \\
& +\frac{1}{2} \sum_{n \neq m=1}^{\infty} \frac{M_{c_{m}}^{i n i t}}{M_{0}} v_{n}\left[v_{m+n}+v_{|m-n|}\right] \frac{\tau_{n} \tau_{m}}{\tau_{m}-\tau_{n}} \\
& \times\left(\tau_{m}\left[1-e^{-t / \tau_{m}}\right]-\tau_{n}\left[1-e^{\left.-t / \tau_{n}\right]}\right)\right)
\end{aligned}
$$

The variance has four contributions. The first is by the longitudinal component of diffusion. The second expresses the interaction of the velocity field and transverse molecular diffusion. The third and fourth terms correct the second term when the particle distribution is initially nonuniform. They describe a contribution of the changing averaged particle velocity resulting from the transverse redistribution. The third term is a direct result of the relaxation of the mean (second term of Eq. (13)). The fourth term accounts for interactions of the higher-order modes. The temporal particle velocity variance is given by

$$
\begin{aligned}
\sigma_{u}^{2}(t)= & \frac{1}{M_{0}} \int_{-\infty}^{\infty} \int_{0}^{d}[v(y)-u(t)]^{2} c(x, y, t) d y d x=\sigma_{v}^{2}+\Delta \sigma_{u}^{2}(t) \\
= & \sum_{n=1}^{\infty} \frac{v_{n}^{2}}{2}+\left[-\left(\sum_{n=1}^{\infty} \frac{v_{n}}{2} \frac{M_{c_{n}}^{i n i t}}{M_{0}} e^{-t / \tau_{n}}\right)^{2}\right. \\
& +\sum_{n=1}^{\infty} \frac{v_{n} v_{2 n}}{4} \frac{M_{c_{n}}^{i n i t}}{M_{0}} e^{-t / \tau_{n}} \\
& \left.+\sum_{m \neq n=1}^{\infty} \frac{\left[v_{m+n}+v_{|m-n|}\right] v_{m}}{4} \frac{M_{c_{n}}^{i n i t}}{M_{0}} e^{-t / \tau_{n}}\right] .
\end{aligned}
$$

$\Delta \sigma_{u}^{2}(t)$ is the deviation of the particle velocity variance from the fluid velocity variance $\left(\sigma_{v}^{2}\right)$ for transverse nonuniform particle distributions. For short times $(t \ll \tau)$, the part of the spreading (15) induced by convection is proportional to time squared and to the variance of the initial particle velocities,

$$
\sigma_{c, x, c o n v}^{2}(t)=2 D_{m o l} t+\sigma_{u}^{2}(0) t^{2}
$$

For uniform particle distributions, two velocity fields with the same velocity variance but with different modal composition produce at early times the same amount of spreading.

In the long-time limit $(t \gg \tau)$, the variance behaves Fickian and is proportional to $\mathrm{t}$,

$$
\sigma_{c, x, \text { disp }}^{2}(t)=2 D_{e f f, \infty} t+\Delta \sigma_{u n i}^{2}+\Delta \sigma_{n o n}^{2} .
$$

As ultimately the particle distribution becomes uniform over the height, only the first two terms of Eq. (15) contribute to the effective asymptotic dispersion coefficient $\left(D_{\text {eff, } \infty}\right)$,

$$
D_{e f f, \infty}=D_{m o l}+\frac{1}{2} \sum_{n=1}^{\infty} v_{n}^{2} \tau_{n} .
$$

For fields with the same velocity variance, those dominated by lower-order modes $\left(v_{n}\right)$ show ultimately more spreading than those dominated by higher-order modes. In Eq. (18), $\Delta \sigma_{u n i}^{2}$ is the constant contribution to the variance resulting from the relaxation of the uniform part of the variance [second term in Eq. (15)] defined as

$$
\Delta \sigma_{u n i}^{2}=-\sum_{n=1}^{\infty} v_{n}^{2} \tau_{n}^{2} \text {. }
$$

$\Delta \sigma_{\text {non }}^{2}$ is a constant contribution to the variance that results from the relaxation of the nonuniform part [third and fourth terms of Eq. (16)] given by

$$
\begin{aligned}
\Delta \sigma_{\text {non }}^{2}= & +\sum_{m \neq n=1}^{\infty} \frac{M_{c_{m}}^{\text {init }}}{M_{0}} \frac{v_{n}\left[v_{m+n}+v_{|m-n|}\right] \tau_{n} \tau_{m}}{2} \\
& -\left(\sum_{n=1}^{\infty} \frac{M_{c_{n}}^{i n i t}}{M_{0}} \frac{v_{n} \tau_{n}}{2}\right)^{2}+\sum_{n=1}^{\infty} \frac{M_{c_{n}}^{i n i t}}{M_{0}} \frac{v_{n} v_{2 n} \tau_{n} \tau_{2 n}}{2} .
\end{aligned}
$$

Figure 4 shows the variance for a uniform initial particle distribution. It shows a relaxation from a $\mathrm{t}^{2}$ behavior for short times towards Fickian behavior $(\sim \mathrm{t})$ for long times. 


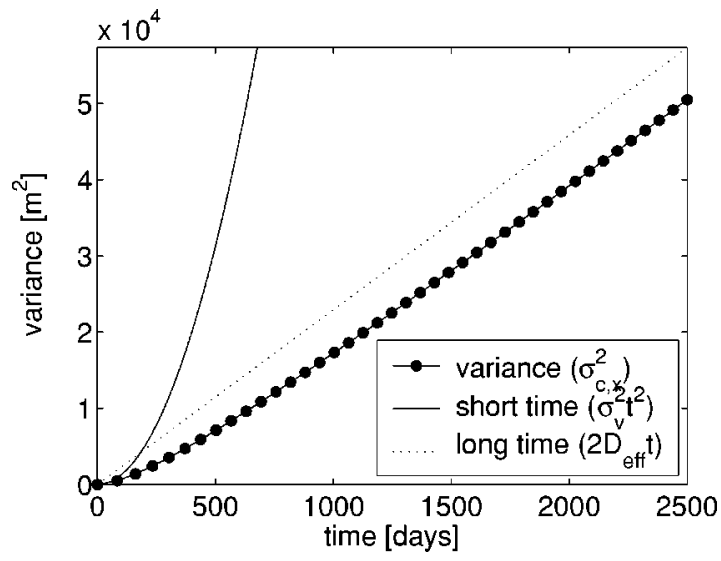

FIG. 4. Relaxation of the variance from convective $\left(\sim t^{2}\right)$ for short times to Fickian behavior $(\sim t)$ for large times.

The shift in the variance $\left[\Delta \sigma_{u n i}^{2}\right.$, Eq. (20)] resulting from the relaxation (Fig. 4) is negative as the variance time derivative monotonously increases towards its asymptotic value $\left(2 D_{\text {eff }, \infty}\right)$.

\section{EVALUATION OF THE UPSCALED RELAXATION MODEL BY CAMACHO}

The laminar flow problem studied by Camacho [16] exhibits a single dominant Fourier scale. He derived an evolution equation for the Taylor flux by summing the relaxation of the modal Taylor fluxes $\left(J_{n}=v_{n} c_{n} / 2\right)$ [27],

$$
\frac{\partial J_{T}}{\partial t}+\frac{J_{T}}{\tau}+v_{0} \frac{\partial J_{T}}{\partial x}+\beta \frac{\partial J_{T}}{\partial x}-D_{m o l} \frac{\partial^{2} J_{T}}{\partial x^{2}}=-\sigma_{v}^{2} \frac{\partial c_{0}}{\partial x} .
$$

Subsequently, he replaced $\tau(\mathrm{x}, \mathrm{t})$ and $\beta(\mathrm{x}, \mathrm{t})$ by the constants $\tau_{\text {eff }}$ and $\beta_{\text {eff }}$ yielding an effective single scale relaxation. Combination of Eqs. (2) and (22) yields a fourth-order PDE for $\mathrm{c}_{0}(\mathrm{x}, \mathrm{t})$.

$$
\begin{aligned}
\frac{\partial c_{0}}{\partial t}+ & v_{0} \frac{\partial c_{0}}{\partial x}-D_{m o l} \frac{\partial^{2} c_{0}}{\partial x^{2}}+\tau_{e f f}\left[\frac{\partial^{2} c_{0}}{\partial t^{2}}\right. \\
& \left.+\left[\left(v_{0}+\beta_{e f f}\right) v_{0}-\sigma_{v}^{2}\right] \frac{\partial^{2} c_{0}}{\partial x^{2}}+\left(2 v_{0}+\beta_{e f f}\right) \frac{\partial^{2} c_{0}}{\partial x \partial t}\right] \\
& =\tau_{e f f}\left[2 D_{m o l} \frac{\partial^{3} c_{0}}{\partial x^{2} \partial t}+\left(2 v_{0}+\beta_{e f f}\right) D_{m o l} \frac{\partial^{3} c_{0}}{\partial x^{3}}-D_{m o l}^{2} \frac{\partial^{4} c_{0}}{\partial x^{4}}\right]
\end{aligned}
$$

Camacho [16] effectively reduced the general multiscale problem to a single scale problem. We investigate the deviations from the full problem by comparing the mean and variance of the approximation with the exact results.

\section{A. Comparison of the mean particle position}

The expression for the mean particle position using the upscaled equation (23) is similar to the full solution [Eq. (13)] but restricted to a single relaxation scale (see Appendix B 1),

$$
\mu_{c, x}^{a p}=x_{0}+v_{0} t+\tau_{e f f}\left(u_{0}-v_{0}\right)\left(1-e^{-t / \tau_{e f f}}\right) .
$$

Equation (24) only matches the mean of the full model for single scale problems or for initially uniform distributions. The convective limit is always correctly described as it follows from the initial conditions. The constant contribution to the Fickian limit originating from the relaxation process can be made to match the exact value by defining the effective relaxation time as

$$
\tau_{\text {eff }}=\sum_{n=1}^{\infty} \frac{\tau_{n} v_{n} \frac{M_{c_{n}}^{\text {init }}}{M_{0}}}{2\left(u_{0}-v_{0}\right)}=\frac{\sum_{n=1}^{\infty} \tau_{n} v_{n} \frac{M_{c_{n}}^{\text {init }}}{M_{0}}}{\sum_{n=1}^{\infty} v_{n} \frac{M_{c_{n}}^{\text {init }}}{M_{0}}} .
$$

Note that this approach fails when the initial distribution is nonuniform and $\mathrm{u}_{0}=\mathrm{v}_{0}$.

\section{B. Comparison of the variance}

The variance calculated from the upscaled equations has again a structure similar to the full expression [Eq. (15)] but restricted to a single scale (see Appendix B 2),

$$
\begin{aligned}
\sigma_{c, x}^{a p, 2}= & 2 D_{m o l} t+2 \sigma_{v}^{2} \tau_{\mathrm{eff}}\left[\tau_{\mathrm{eff}}\left(e^{-t / \tau_{\mathrm{eff}}}-1\right)+t\right]+2 \tau_{\mathrm{eff}} \beta_{\mathrm{eff}}\left(u_{0}-v_{0}\right) \\
& \times\left[\tau_{\mathrm{eff}}\left(e^{-t / \tau_{\mathrm{eff}}}-1\right)+t e^{-t / \tau_{\mathrm{eff}}}\right]-\left\{\tau _ { \mathrm { eff } } ( u _ { 0 } - v _ { 0 } ) \left[e^{-t / \tau_{\mathrm{eff}}}\right.\right. \\
& -1]\}^{2}
\end{aligned}
$$

For uniform initial distributions, the third and fourth terms on the right-hand side disappear. As only a single effective relaxation process is present in the approximation, it is only exact for single scale problems. The short-time limit is again exact. In the long-time limit $(t \gg \tau)$, the approximation reduces to

$$
\sigma_{c, x, d i s p}^{a p, 2}=2\left(D_{m o l}+\sigma_{v}^{2} \tau_{\text {eff }}\right) t .
$$

If we set the effective relaxation time $\left(\tau_{e f f}\right)$ equal to the so-called standard relaxation time $\tau_{\text {std }}$ (see Camacho [16]),

$$
\tau_{e f f}=\tau_{s t d} \equiv \frac{1}{2 \sigma_{v}^{2}} \sum_{n=1}^{\infty} v_{n}^{2} \tau_{n}=\frac{\sum_{n=1}^{\infty} v_{n}^{2} \tau_{n}}{\sum_{n=1}^{\infty} v_{n}^{2}},
$$

we also match the exact variance in the long-time limit (18). However, Sec. X will show that this definition does not give an accurate approximation for intermediate times. For an initially nonuniform distribution, the situation is more complex. In the convective limit, the upscaled variance reads

$$
\sigma_{c, x, \text { conv }}^{a p, 2}=2 D_{m o l} t+2 \sigma_{v}^{2} t^{2}-\beta_{\text {eff }}\left(u_{0}-v_{0}\right) t^{2}-\left(u_{0}-v_{0}\right)^{2} t^{2} .
$$

This expression does not have any information on the initial particle velocity variance which drives the variance evolution in the convective limit. However, we can define parameter $\beta_{\text {eff }}$ to introduce this information in such a way that the short-time limit is again exact,

$$
\beta_{e f f}=\frac{\sigma_{v}^{2}-\sigma_{u, i n i t}^{2}-\left(u_{0}-v_{0}\right)^{2}}{u_{0}-v_{0}}
$$


Note that as observed before in the discussion of the mean, in the case $\mathrm{u}_{0}=\mathrm{v}_{0}$ the approach above fails. The upscaled equations simply cannot handle this situation. In the long-time limit $(t \gg \tau)$, the variance shows the correct Fickian behavior,

$$
\begin{aligned}
\sigma_{c, x, d i s p}^{a p, 2}= & 2\left(D_{m o l}+\sigma_{v}^{2} \tau_{\text {eff }}\right) t-\left[2 \sigma_{v}^{2} \tau_{\text {eff }}^{2}+2 \tau_{\text {eff }}^{2} \beta_{\text {eff }}\left(u_{0}-v_{0}\right)\right. \\
& \left.+\tau_{\text {eff }}^{2}\left(u_{0}-v_{0}\right)^{2}\right] .
\end{aligned}
$$

Ultimately, the growth of the variance is independent of the initial distribution. The relaxation constant, however, is different, since the relaxation process has evolved in a different way. To match the approximation with the exact variance in the Fickian limit, the uniform definition for the relaxation time can be applied. The constant contribution due to the relaxation process, however, cannot be matched.

\section{Relaxation time considerations}

For nonuniform initial conditions, the effective relaxation time has to be tuned to the initial distribution (25) to obtain a match for the mean for long times. Turning to the effective dispersion coefficient in the Fickian limit, we can match the upscaled expression to the exact relationship by an appropriate definition of the effective relaxation time (28). However, as may be clear from the physics, this definition is based on the fluid velocity profile and is unrelated to the initial distribution. The upscaled model can only match one of the two conditions.

\section{GENERALIZED TELEGRAPH EQUATION}

The fourth-order upscaled PDE Eq. (23) covers the whole spectrum from cases where (longitudinal) molecular diffusion dominates the spreading to cases where the transverse variation in the velocity field dominates the spreading. If the contribution by longitudinal molecular diffusion to the behavior of the fourth-order model can be neglected, the parabolic fourth-order upscaled equation reduces to a secondorder hyperbolic generalized Telegraph equation,

$$
\begin{gathered}
\tau_{e f f} \frac{\partial^{2} c_{0}}{\partial t^{2}}+\frac{\partial c_{0}}{\partial t}+v_{0} \frac{\partial c_{0}}{\partial x}+\tau_{e f f}\left(2 v_{0}+\beta_{e f f}\right) \frac{\partial^{2} c_{0}}{\partial x \partial t} \\
-\tau_{e f f}\left[\sigma_{v}^{2}-\left(v_{0}+\beta_{e f f}\right) v_{0}\right] \frac{\partial^{2} c_{0}}{\partial x^{2}}=0 .
\end{gathered}
$$

This equation describes the same mean and variance as the fourth-order model if the contribution of the spreading caused by longitudinal molecular diffusion is ignored.

\section{A. Concentration profiles}

Unfortunately, the fourth-order upscaled PDE Eq. (23) does not have an easily analyzable analytical solution. In contrast, for initial conditions $c(x, y, 0)=H\left(x-x_{0}\right)$ and $\partial_{t} c_{0}(x, 0)=0$, the solution to the Telegraph model Eq. (32) reads for $x \in(-\infty, \infty)$, see [18],

$$
c(x, t)= \begin{cases}H(\chi-\Gamma)\left[D e^{-\chi} I_{0}\left\{\sqrt{\chi^{2}-\Gamma^{2}}\right\}+\frac{e^{-\Gamma}}{2}+\frac{\Gamma}{2} \int_{\Gamma}^{\chi} \frac{e^{-\tilde{\xi}}}{\sqrt{\tilde{\xi}^{2}-\Gamma^{2}}} I_{1}\left\{\sqrt{\left.\tilde{\xi}^{2}-\Gamma^{2}\right\}} d \tilde{\xi}\right],\right. & x>v_{0} t \\ 1+H(\chi-\Gamma)\left[D e^{-\chi} I_{0}\left\{\sqrt{\chi^{2}-\Gamma^{2}}\right\}-\frac{e^{-\Gamma}}{2}-\frac{\Gamma}{2} \int_{\Gamma}^{\chi} \frac{e^{-\tilde{\xi}}}{\sqrt{\tilde{\xi}^{2}-\Gamma^{2}}} I_{1}\left\{\sqrt{\tilde{\xi}^{2}-\Gamma^{2}}\right\} d \tilde{\xi}\right], & x<v_{0} t\end{cases}
$$

with

$$
\begin{aligned}
& \chi=\frac{2 \psi}{\varepsilon^{2}+4 \psi \varphi}\left(t\left[1-\frac{v_{0} \varepsilon}{2 \psi}\right]+x \frac{\varepsilon}{2 \psi}\right), \\
& \Gamma=\frac{\left|x-v_{0} t\right|}{\sqrt{\varepsilon^{2}+4 \psi \varphi}}, \quad D=\frac{-\varepsilon}{2 \sqrt{\varepsilon^{2}+4 \psi \varphi}},
\end{aligned}
$$

and

$$
\varphi=\tau_{\text {eff }}, \quad \varepsilon=+\beta_{\text {eff }} \tau_{\text {eff }}, \quad \psi=\sigma_{v}^{2} \tau_{\text {eff }} .
$$

For the velocity field shown in Fig. 1, we compare the concentration profiles by the fourth-order model (23), the Telegraph equation (32), and the height averaged concentration profile by the full 2D model (1). Both upscaled models show nearly identical concentration profiles during the relaxation from convective to Fickian behavior [Figs. 5, 6(a), and $6(b)]$, even for relatively large values for the molecular diffusion [Fig. 6(b)]. For short times, the hyperbolic part $\left(\tau_{e f f} \partial_{t}^{2} c_{0}\right)$ in the Telegraph model dominates over the parabolic part $\left(\partial \mathrm{c}_{0} / \partial \mathrm{t}\right)$ and its concentration profile shows two shocks, typical for a second-order hyperbolic equation. Even though the profiles of the upscaled models seem to give a poor representation of the exact profile [Fig. 6(a)], they match the exact solution up to the second moment. In the long-term limit, both upscaled models demonstrate Fickian behavior, characterized by the typical S-shaped concentration profile, and give an excellent match with the exact solution [Fig. 6(b)]. Note that the propagation speed of the Telegraph model is constant and finite, contrary to the fourth-order model and classical convection diffusion equation [28]. 


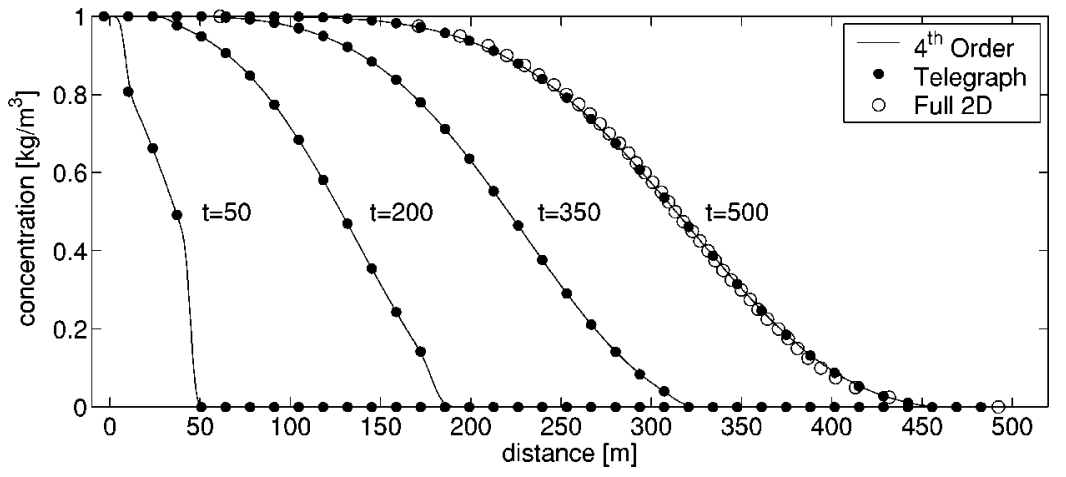

FIG. 5. Evolution of the concentration profiles of Camacho's fourth-order model and the generalized Telegraph equation (for $\mathrm{D}_{m o l}=1.25$ $\left.\times 10^{-4} \mathrm{~m}^{2} \mathrm{~d}^{-1}\right)$. For $\mathrm{t}=500 \mathrm{~d}$, the profile obtained from a full $2 \mathrm{D}$ random-walk simulation is shown for comparison.

\section{PHYSICS-REVERSED FLOW}

Now consider the case where after a time $\mathrm{t}=\mathrm{t}_{r}$ we reverse the flow direction. Assuming convective transport only, the process is fully reversible and the particles are transported back to their original positions, see Fig. 7(a).

\section{A. Reversal of flow and relaxation}

If we reverse the flow and include molecular diffusion, the relaxation process starts all over again. However, the particle distribution at the time of reversal is shaped by the convection dispersion process. As discussed above, each particle velocity in this process is correlated over a short time.
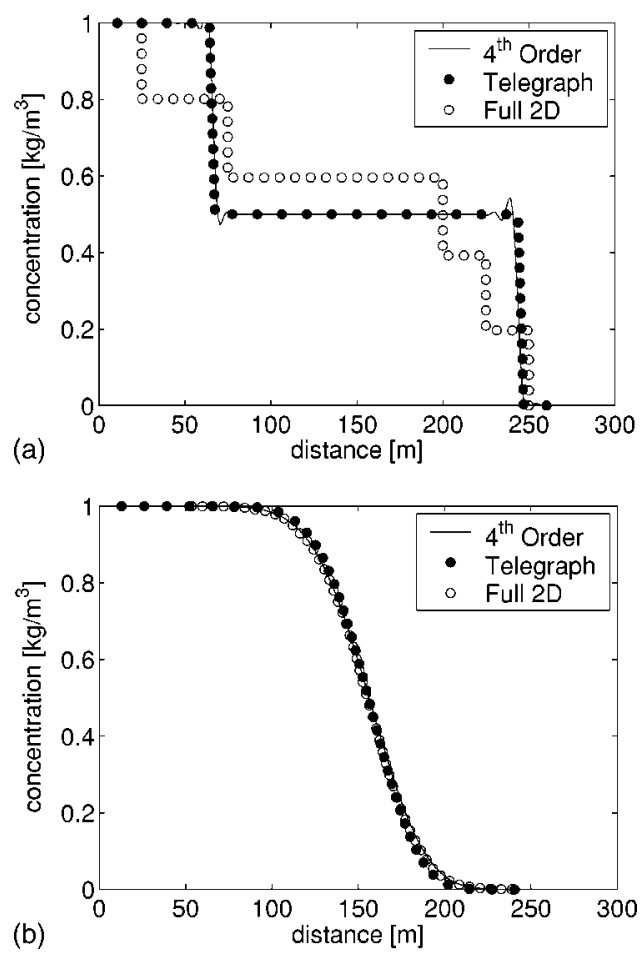

FIG. 6. Comparison of the concentration profiles of the full 2D, Camacho's fourth-order, and the Telegraph model at time $t=25 \mathrm{~d}$. (a) The concentration profile in the convective limit for $\mathrm{D}_{m o l}=2.5$ $\times 10^{-9} \mathrm{~m}^{2} \mathrm{~d}^{-1}$. (b) The concentration profile in the long-time Fickian limit for $\mathrm{D}_{m o l}=6.25 \times 10^{-4} \mathrm{~m}^{2} \mathrm{~d}^{-1}$. Note that the (Gibbs) overshoots in the fourth-order model are caused by the third-order upwind scheme that is used for the spatial discretization.
As a result, initially particles return longitudinally along the same path as they arrived from. Hence the transport process demonstrates a (partially) reversible behavior. The variance of the height-averaged particle positions decreases as the particles turn back along their original paths. Due to transverse diffusion, the velocity of a particle becomes in time less correlated to the velocity history of its forward movement. As a result, convective dispersion takes over and the variance once again increases monotonously. The process is identical to the original relaxation process. Consequently, the same relaxation time characterizes the interaction process of the reversed velocity field and molecular diffusion to form a dynamic equilibrium exhibiting a Fickian behavior. Moreover, this (relaxation) time is independent of the moment in time at which we reverse the flow.

Consider a single scale relaxation process with relaxation time $\tau$. We distinguish three cases based on the ratio of dimensionless reversal time, $t_{r D}=t_{r} / \tau$. For $t_{r D} \ll 1$, the particles exhibit a fully correlated behavior similar to pure convection [Fig. 7(a)]. In contrast, if $t_{r D} \gg 1$, the transport process is fully relaxed at the time of flow reversal [Fig. 7(c)]. For intermediate times $t_{r D}$, the particles, at the time of flow reversal, will be significantly correlated to their original streamlines and each particle velocity, at the time of reversal, will be correlated to the initial particle velocity in time. Consequently, the velocity profile may be clearly visible in the particle cloud [Fig. 7(b)]. Although the reversibility may be much more significant and visible for small $t_{r D}<1$, partial reversibility, however small, is observed for all values of $t_{r D}$. In general, the relaxation process may evolve over multiple scales. At times smaller than the relaxation time of the largest scale, the various modes will generally not be in the same state of relaxation.

\section{B. Evolution of the variance}

If we reverse the flow, two combined processes contribute to the variance. The first process expresses the correlation between forward and reversed velocities of a particle and causes a decrease in the variance. The second is a new relaxation process identical to unidirectional relaxation, but with the particle distribution at reversal as initial distribution. Initially, the first process dominates and the reversed velocity of a particle is completely correlated to its last forward velocity. Consequently the time derivative of the variance changes sign [Fig. 8(b)], which we experience, even in the 

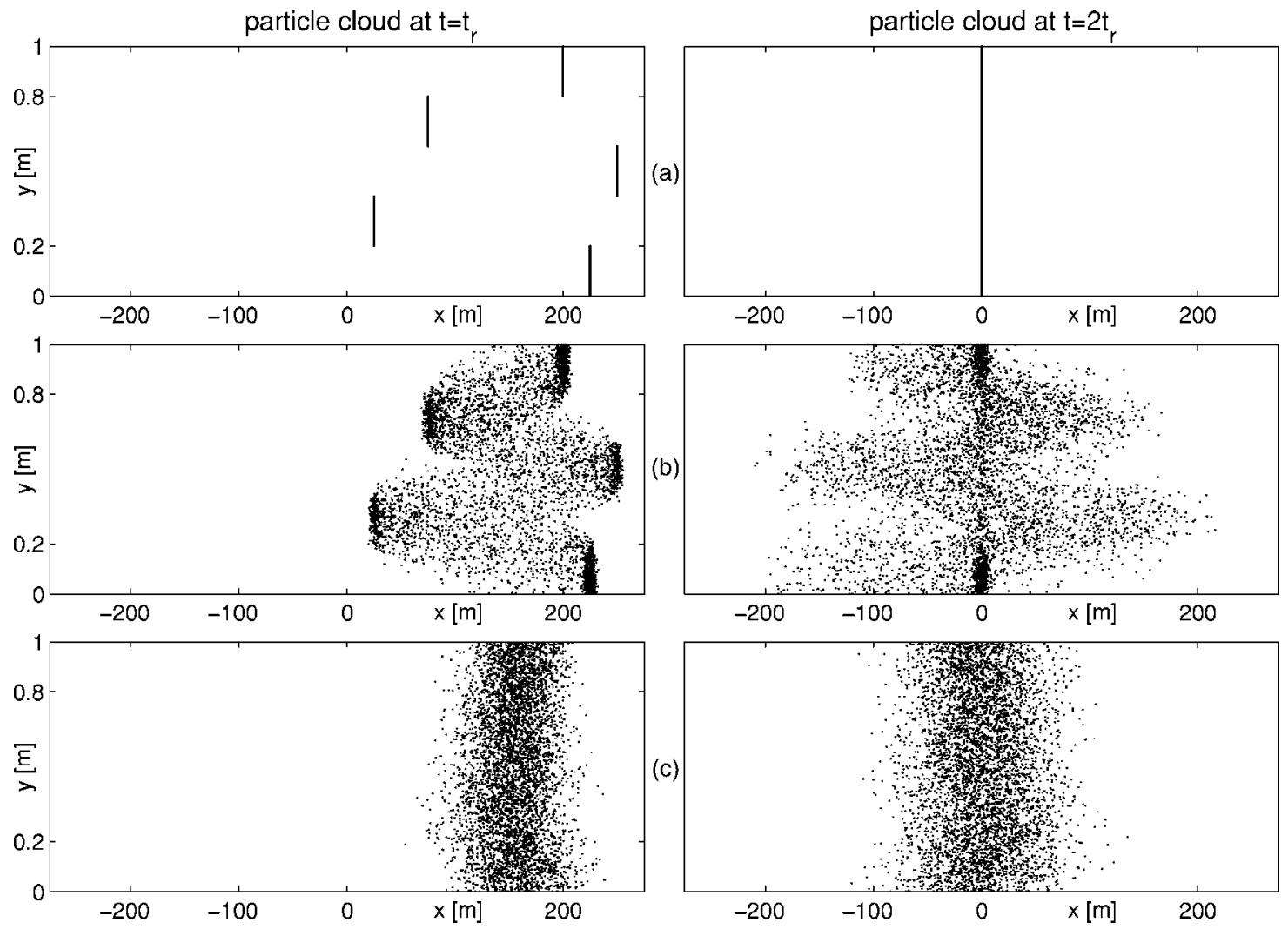

FIG. 7. Particle clouds belonging to the five-layer velocity field (Fig. 1) at the moment of flow reversal $\left(t=t_{r}\right)$ and at time $\left(t=2 t_{r}\right)$ for three different relaxation regimes. (a) The convective limit $\left(\mathrm{D}_{\text {mol }}=0 \mathrm{~m}^{2} \mathrm{~d}^{-1}\right)$. (b) Intermediate situation $\left(\mathrm{D}_{\text {mol }}=3 \times 10^{-5} \mathrm{~m}^{2} \mathrm{~d}^{-1}\right)$. $(\mathrm{c})$ The Fickian limit $\left(\mathrm{D}_{\text {mol }}=6.3 \times 10^{-4} \mathrm{~m}^{2} \mathrm{~d}^{-1}\right)$.

Fickian limit, as "partial" reversibility. With time a particle velocity loses its correlation with the forward velocities, starting with the short-time correlations (or small scales). At a certain moment, the two processes counterbalance each other, marked by the point at which the variance reaches its minimum [Fig. 8(a)] and its time derivative passes zero [Fig. $8(b)]$. From this point on, the second process dominates and relaxes to the same Fickian behavior as for unidirectional flow while the first process relaxes to a constant value. In the convective limit, the velocity of each particle is fully correlated (or constant) in time and the first process dominates up to the point that the time after reversal equals the reversal time [Fig. 8(a), $\tau=25000]$.

\section{MOMENT ANALYSIS FOR FLOW INCLUDING REVERSAL OF DIRECTION}

Here we analyze the spatial moments belonging to the $2 \mathrm{D}$ uCDifE for flow including reversal. The evolution expressions for the $k$ th moments [29] $M_{c_{0}, x, k}^{r e v}(t)$ and $E_{c_{n}, x, k}^{r e v}(t)$ after flow reversal are similar to expressions (8) and (9), respectively, with the signs of the modal velocities changed.

\section{A. Transverse particle distribution and mean particle position}

Flow reversal does not affect the way transverse diffusion redistributes the particles in the vertical direction. Hence, flow reversal does not affect the zeroth moment of the higher-order modes and is given by Eq. (11). The mean particle velocity is directly related to the transverse distribution and only changes sign when the flow direction is reversed. The mean particle position for $t>t_{r}$ reads

$$
\begin{aligned}
\mu_{c, x}^{r e v}(t)= & x_{0}+v_{0}\left(2 t_{r}-t\right)+\frac{1}{2} \sum_{n=1}^{\infty} \tau_{n} v_{n} \frac{M_{c_{n}}^{i n i t}}{M_{0}} \\
& \times\left[e^{-t / \tau_{n}}-2 e^{-t_{r} / \tau_{n}}+1\right], \quad t>t_{r} .
\end{aligned}
$$

In the convective limit, the mean is fully reversible, $\mu_{c, x, \text { conv }}^{r e v}=x_{0}+u_{0}\left(2 t_{r}-t\right)$. In the Fickian limit $(t>\tau)$, the vertical particle distribution is degenerated to a uniform distribution and the mean particle position reads

$$
\begin{aligned}
\mu_{c, x, \text { disp }}^{r e v}(t)= & \mu_{c, x, \text { uni }}^{r e v}(t)+\Delta \mu_{\text {relax }}^{\text {rev }} \\
& =\left[x_{0}+v_{0}\left(2 t_{r}-t\right)\right] \\
& +\left[\sum_{n=1}^{\infty} \frac{\tau_{n} v_{n}}{2} \frac{M_{c_{n}}^{\text {init }}}{M_{0}}\left(1-2 e^{\left.-t_{r} / \tau_{n}\right)}\right] .\right.
\end{aligned}
$$

The deviation of the particle mean from the fluid mean, expressed by the relaxation constant $\Delta \mu_{\text {relax }}^{r e v}$, is a function of the reversal time $t_{r}$ and converges to $\Delta \mu_{\text {relax }}$ (14) for $t_{r} \gg \tau$.

\section{B. The variance of the averaged concentration}

The exact variance for uniform initial distributions is given by (see appendix A 2) 

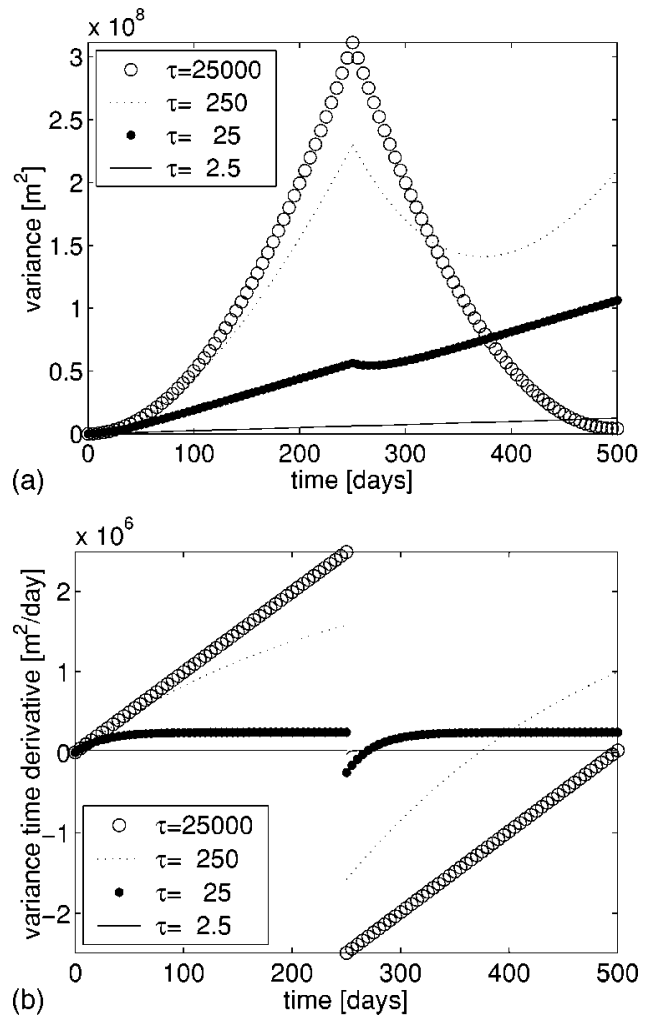

FIG. 8. (a) Typical example of the variance for flow including reversal of direction at time $t=250$ days and (b) the corresponding time derivative for four values of the relaxation time $\tau$ $=(2.5,25,250$, and 25000$)$ days.

$$
\begin{aligned}
\sigma_{c, x, \text { rev }, \text { uni }}^{2}(t)= & 2 D_{\text {mol }} t+\sum_{n=1}^{\infty} \tau_{n} v_{n}^{2} \times\left[\left[t_{r}+\tau_{n}\left(e^{-t_{r} / \tau_{n}}-1\right)\right]\right. \\
& +\left[\left(t-t_{r}\right)+\tau_{n}\left(e^{-\left(t-t_{r}\right) / \tau_{n}}-1\right)\right] \\
& \left.+\left[\tau_{n}\left(e^{t_{r} / \tau_{n}}-1\right) e^{-t / \tau_{n}}+\tau_{n}\left(e^{-t_{r} / \tau_{n}}-1\right)\right]\right] .
\end{aligned}
$$

The contribution by the longitudinal component of molecular diffusion is independent of the flow direction. The contribution by convection and transverse diffusion has three subterms. The first expresses the value of the variance at the time of reversal. The second represents a new relaxation process, identical to the initial one. The third term is a "demixing" or reversibility term expressing the effect of flow reversal on the variance induced by the forward convectiondispersion process. It is increasingly negative for $t>t_{r}$ and asymptotically reaches a constant once correlation with the flow before reversal is lost. For an arbitrary initial distribution, the exact variance after flow reversal reads (Appendix A 2)

$$
\begin{aligned}
\sigma_{c, x, \text { rev }}^{2}(t)= & \sigma_{c, x, \text { rev }, \text { uni }}^{2}(t)-\left(\frac { 1 } { 2 } \sum _ { n = 1 } ^ { \infty } \tau _ { n } v _ { n } \frac { M _ { c _ { n } } ^ { \text { init } } } { M _ { 0 } } \left[e^{-t / \tau_{n}}-2 e^{-t_{r} / \tau_{n}}\right.\right. \\
& +1])^{2}+\frac{1}{2} \sum_{n=1}^{\infty} \frac{M_{c_{n}}^{i n i t}}{M_{0}} v_{n} v_{2 n}\left(2 t_{r} \tau_{n}\left[e^{-t / \tau_{n}}-e^{-t_{r} / \tau_{n}}\right]\right. \\
& \left.-t \tau_{n} e^{-t / \tau_{n}}+\tau_{n}^{2}\left[1-e^{-t / \tau_{n}}\right]\right)
\end{aligned}
$$

$$
\begin{aligned}
& +\frac{1}{2} \sum_{m, n=1(m \neq n)}^{\infty} \frac{M_{c_{m}}^{\text {init }}}{M_{0}} v_{n}\left[v_{m+n}+v_{|m-n|}\right] \frac{\tau_{n} \tau_{m}}{\tau_{m}-\tau_{n}} \\
& \times\left(\tau_{m}\left[1-e^{-t / \tau_{m}}\right]-\tau_{m}+2 \tau_{n}\left[e^{-t_{r} / \tau_{n}}-e^{-t / \tau_{m}}\right]\right. \\
& \left.\left.+\tau_{n} e^{t / \tau_{n}\left[2 e^{-t} / \tau_{m}\right.}-e^{-t_{r} / \tau_{n}}\right] e^{-t / \tau_{n}}\right)
\end{aligned}
$$

The additional contribution to the variance only depends on the reversal time $t_{r}$ if the distribution has not yet relaxed to a uniform distribution before reversal. Taking the time derivative of Eq. (39) shows that for any value of $\tau$, immediately upon flow reversal the dispersive contribution to the variance is negative,

$$
\lim _{t \downarrow t_{r}}\left(\frac{\partial \sigma_{c, x, r e v}^{2}}{\partial t}-2 D_{m o l}\right)=-\lim _{t \uparrow t_{r}}\left(\frac{\partial \sigma_{c, x}^{2}}{\partial t}-2 D_{m o l}\right) .
$$

In the convective limit $\left(t, t_{r} \ll \tau\right)$, no relaxation takes place and the variance by the convective-dispersive part displays fully reversible behavior, reducing to zero at $t=2 t_{r}$,

$$
\sigma_{c, x, r e v, c o n v}^{2}(t)=\sigma_{u}^{2}(0)\left(t-2 t_{r}\right)^{2},
$$

where $\sigma_{u}^{2}$ is given by Eq. (16). In the Fickian limit $(t \gg \tau)$, the variance takes the form

$$
\sigma_{c, x, r e v, d i s p}^{2}(t)=2 D_{e f f, \infty} t+\Delta \sigma_{\text {uni,rev }}^{2}+\Delta \sigma_{\text {non,rev }}^{2} .
$$

The asymptotic dispersion coefficient $D_{e f f, \infty}$ is independent of the flow direction and is given by Eq. (19). The constant contribution to the variance resulting from the relaxation process for initially uniform particle distribution, $\Delta \sigma_{\text {uni,rev }}^{2}$, varies with the reversal time,

$$
\Delta \sigma_{\text {uni,rev }}^{2}=-\sum_{n=1}^{\infty} \tau_{n}^{2} v_{n}^{2}\left(3-2 e^{-t_{r} / \tau_{n}}\right) .
$$

Its magnitude is bounded between $\Delta \sigma_{u n i}^{2}$ (for $t_{r} \ll \tau$ ) and $3 \Delta \sigma_{\text {uni }}^{2}$ (for $\left.t_{r} \gg \tau\right)$. The constant contribution to the variance resulting from the relaxation of a transverse nonuniform to a transverse uniform particle distribution $\Delta \sigma_{\text {non,rev }}^{2}$ reads

$$
\begin{aligned}
\Delta \sigma_{\text {non, rev }}^{2}= & -\left(\frac{1}{2} \sum_{n=1}^{\infty} \tau_{n} v_{n} \frac{M_{c_{n}}^{\text {init }}}{M_{0}}\left[1-2 e^{\left.-t_{r} / \tau_{n}\right]}\right)^{2}\right. \\
& +\frac{1}{2} \sum_{n=1}^{\infty} \frac{M_{c_{n}}^{i n i t}}{M_{0}} v_{n} v_{2 n} \tau_{n}\left[\tau_{n}-2 e^{\left.-t_{r} / \tau_{n} t_{r}\right]}\right. \\
& +\frac{1}{2} \sum_{m \neq n=1}^{\infty} \frac{M_{c_{m}}^{i n i t}}{M_{0}} v_{n}\left[v_{m+n}+v_{|m-n|}\right] \\
& \times\left(\tau_{n} \tau_{m}+2 \tau_{n} \frac{e^{-t_{r} / \tau_{n}}-e^{-t_{r} / \tau_{m}}}{\tau_{n}-\tau_{m}}\right)
\end{aligned}
$$

If the transverse particle distribution has relaxed to uniformity before flow reversal, $\Delta \sigma_{\text {non, rev }}^{2}$ equals $\Delta \sigma_{\text {non }}^{2}(21)$.

\section{PERIODIC FLOW REVERSAL}

If we keep reversing the flow each time thas increased by $\mathrm{t}_{r}$, the time derivative of the variance asymptotically turns 
periodic as well [Fig. 9(a)]. Ultimately the periodic growth of the variance becomes linear in time even if the variance itself does not relax in a period $t_{r}$ [Fig. 9(b)]. Each reversal a new (but identical) relaxation process starts while a second process expresses the correlations with the previous reversal cycles. After sufficient time has passed, the velocity of a particle in the $n$th reversal cycle is no longer correlated to the velocity in the first forward period. The time to lose correlation is again related to the relaxation time. After this time has passed, a particle experiences statistically the same velocity correlation in each subsequent reversal period.

\section{A. Mathematical derivation}

Moment analysis shows that the time derivative of the variance becomes periodic when the flow is reversed periodically. Since nonuniform initial distributions relax ultimately to a uniform distribution, we limit the discussion to uniform initial particle distributions $\left(M_{c_{n}, 0}^{\text {init }}=0\right)$. The time derivative of the variance in the $\left(\mathrm{N}_{R}\right)$ th reversal cycle reads (Appendix C)

$$
\begin{aligned}
\frac{\partial \sigma_{c, x}^{2}}{\partial t}\left(\tilde{t} ; N_{r}, t_{r}\right)= & 2 D_{m o l}+\sum_{n=1}^{\infty} v_{n}^{2} \tau_{n} \times\left[1+(-1)^{N_{r}} e^{-\left(\tilde{t}+N_{r} t_{r}\right) / \tau_{n}}\right. \\
& \left.-2 \frac{e^{-\left(\tilde{t}-t_{r}\right) / \tau_{n}}+(-1)^{N_{r}} e^{-\left(\tilde{T}+N_{r} t_{r}\right) / \tau_{n}}}{e^{t_{r} / \tau_{n}}+1}\right]
\end{aligned}
$$

where $\tilde{t}$ is the time since the last flow reversal. For $\left(N_{r}\right.$ $\rightarrow \infty$ ), this expression (45) converges to

$$
\begin{aligned}
\frac{\partial \sigma_{c, x, l i m}^{2}}{\partial t}\left(\tilde{t} ; t_{r}\right)= & \lim _{N_{r} \rightarrow \infty} \frac{\partial \sigma_{c, x}^{2}}{\partial t}\left(\widetilde{t} ; N_{r}, t_{r}\right) \\
& =2 D_{m o l}+\sum_{n=1}^{\infty} v_{n}^{2} \tau_{n}\left[1-2 \frac{e^{-\left(\tilde{t}-t_{r}\right) / \tau_{n}}}{e^{t, / \tau_{n}}+1}\right] .
\end{aligned}
$$

Thus even if the variance does not relax in a period $\mathrm{t}_{r}$ $\left(t_{r}<\tau\right)$, the growth of the variance relaxes if we keep on reversing the flow each time $t$ has increased with $t_{r}$. The net cyclic dispersion coefficient $\mathrm{D}_{\text {cycle }}$ for $N_{r} \rightarrow \infty$ follows by integration of Eq. (46) over a cycle $\left(\tilde{t} \in\left[0, t_{r}\right]\right)$ and division of the result by $\left(2 t_{r}\right)$,

$$
\begin{aligned}
D_{\text {cycle }}= & \frac{1}{2 t_{r}} \int_{0}^{t_{r}} \frac{\partial \sigma_{c, x, \lim }^{2}}{\partial t}\left(\tilde{t} ; t_{r}\right) d \tilde{t} \\
& =D_{m o l}+\frac{1}{2} \sum_{n=1}^{\infty} v_{n}^{2} \tau_{n}\left[1-2 \frac{\tau_{n}\left(e^{t_{r} / \tau_{n}}-1\right)}{t_{r}\left(e^{t_{r} / \tau_{n}}+1\right)}\right] .
\end{aligned}
$$

Figure 10 shows the normalized modal cyclic dispersion coefficient [term between square brackets in Eq. (47)]. It increases monotonously from 0 for the limit of $\mathrm{t}_{r} / \tau_{n}$ to zero towards 1 for the limit of $\mathrm{t}_{r} / \tau_{n}$ to infinity. The corresponding expression of the variance for repetitive flow reversal reads
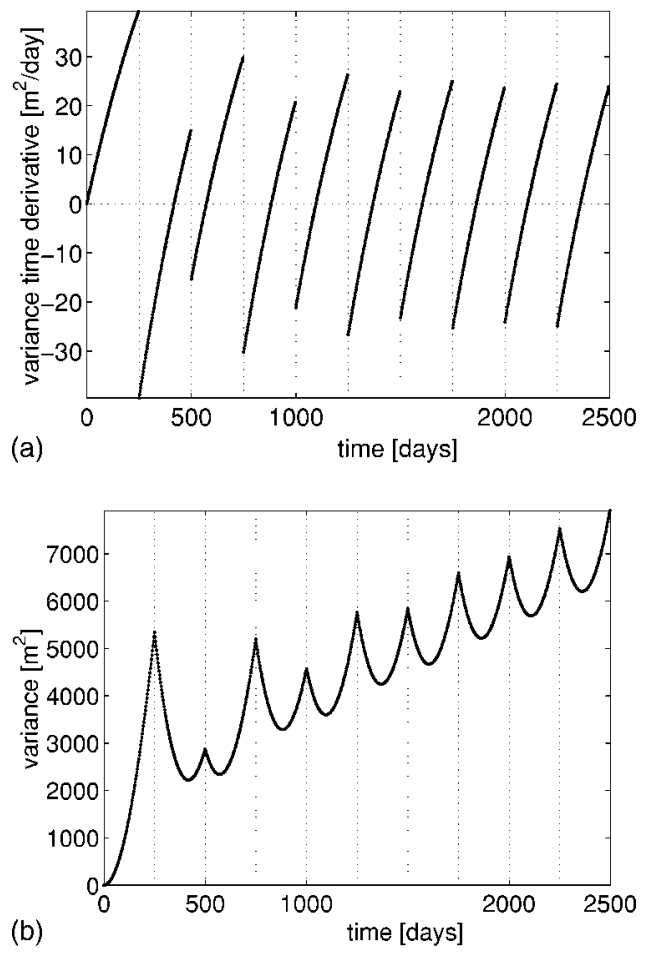

FIG. 9. (a) Convergence of the time derivative of the variance to periodic behavior for periodic reversal. (b) The corresponding evolution of the variance.

$$
\begin{aligned}
\sigma_{c, x}^{2}\left(t ; N_{r}, t_{r}\right)= & 2 D_{m o l} t+\sum_{n=1}^{\infty} v_{n}^{2} \tau_{n}^{2} \times\left(\frac{t}{\tau_{n}}\right. \\
& +2 \sum_{k=0}^{N_{r}} '(-1)^{N_{r}-k}\left(e^{-\left(t-k t_{r}\right) / \tau_{n}}-1\right) \\
& \left.-\sum_{k=1}^{N_{r}}(-1)^{k}\left[4\left(N_{r}-k\right)+2\right]\left(e^{-k t_{r} / \tau_{n}}-1\right)\right) .
\end{aligned}
$$

The sums in Eq. (48) increase linearly with the reversal cycle ("time") when the time exceeds the relaxation time [see also Fig. 9(b)]. Figure 11 demonstrates the effect of a decreasing reversal time on the variance.

\section{SEPARATION OF SCALES}

\section{A. Multiple scale treatment}

For multiscale velocity fields, the fourth-order model is approximate. To improve its accuracy, there are two ways to account for the changing interaction of the scales present in the full problem over time. We can optimize the effective relaxation time or apply scale separation. We discuss their relative merit as a function of scale distribution and the observation time. To demonstrate this, consider a bimodal velocity field $\mathrm{v}(\mathrm{y})$,

$$
v(y)=v_{n_{1}} \cos \left(\frac{n_{1} \pi}{d} y\right)+v_{n_{2}} \cos \left(\frac{n_{2} \pi}{d} y\right) .
$$


We define a dimensionless observation time as $t_{\text {obs }}=t / \tau_{1}$, and the closely related modal observation time of mode $\mathrm{n}$ as $t_{n, o b s}=t / \tau_{n}=n^{2} t / \tau_{1}$. In this section, we restrict ourselves to uniform initial particle distributions.

\section{B. Effective relaxation time}

Let both modes in Eq. (49) describe a significant relaxation with respect to the observation time. Take, for example, modes $\mathrm{n}_{1}=1$ with $\tau_{1}=1500 \mathrm{~d}$ and $\mathrm{n}_{2}=5$ with $\tau_{1} / \tau_{2}=25$ and $\mathrm{t}=50$. Figure 12 shows that the variance by the fourth-order model matches the exact variance, using the relaxation time as fit parameter $\left(\tau_{f i t}=130\right)$. In contrast, the standard definition (28) overestimates the relaxation time $\left(\tau_{\text {std }}=780\right)$ and erroneously predicts the variance. To provide an improved $a$ priori estimate of the relaxation time, consider the evolution equation for the approximate variance (B5) for uniform conditions $\left(\mathrm{u}_{0}=\mathrm{v}_{0}\right)$. Replacing the approximate variance $\left(\sigma_{a p p, x}^{2}\right)$ by the exact variance for unidirectional flow $\left(\sigma_{c, x}^{2}\right)(15)$ and rearranging terms gives the evolution of the effective relaxation as a function of time,

$$
\tau_{e f f}(t)=\frac{\frac{\partial \sigma_{a p p, x}^{2}(t)}{\partial t}-2 D_{m o l}}{2 \tau \sigma_{v}^{2}-\frac{\partial^{2} \sigma_{a p p, x}^{2}(t)}{\partial t^{2}}}=\frac{\sum_{n=1}^{\infty} v_{n}^{2} \tau_{n}\left(1-e^{-t / \tau_{n}}\right)}{\sum_{n=1}^{\infty} v_{n}^{2}\left(1-e^{-t / \tau_{n}}\right)}
$$

This relaxation time matches $\tau_{\text {std }}(28)$ in the Fickian limit and equals the harmonic, modal velocity weighted average of the modal relaxation times for short times,

$$
\lim _{t \downarrow 0} \tau_{e f f}(t)=\frac{\sum_{n=1}^{\infty} v_{n}^{2}}{\sum_{n=1}^{\infty} v_{n}^{2} / \tau_{n}} .
$$

This explains why $\tau_{s t d}(28)$ overestimates the relaxation time for short times. Let us now define $\tau_{a v g}$ as the average of $\tau_{\text {eff }}(\mathrm{t})$ over the time domain $\left[\mathrm{t}_{\text {min }}, \mathrm{t}_{\text {max }}\right]$ of interest,

$$
\tau_{a v g}=\frac{1}{t_{\max }-t_{\min }} \int_{t_{\min }}^{t_{\max }} \tau_{e f f}(t) d t .
$$

Using this relaxation time $\tau_{\text {avg }}$ in the approximate model yields a very good fit with the exact variance Fig. 12.

\section{Separation of scales}

Next we study a two-scale problem (e.g., $\mathrm{n}_{1}=1, \mathrm{n}_{2}=100$ ) and consider observation times in between the two modal relaxation times. Figure 13 shows that the standard relaxation time, Eq. (28), overestimates the variance. Equation (28) describes an averaged relaxation of both scales, while only the larger scale still undergoes a relaxation for the time scale considered here. With scale separation, we put the small relaxed scale $\left(\mathrm{n}_{2}\right)$ together with the molecular diffusion in a microscale dispersion coefficient [30],

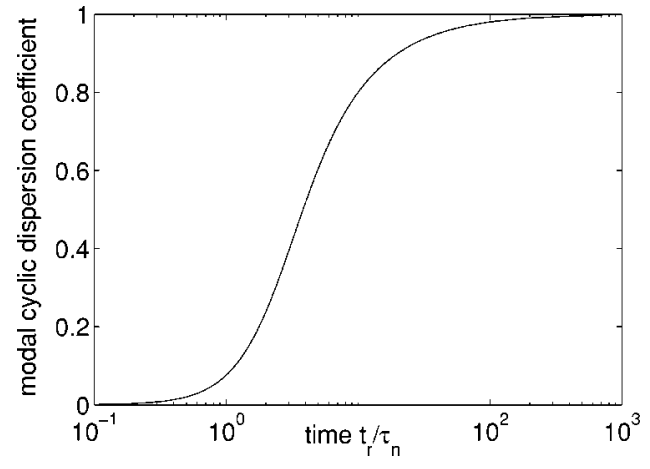

FIG. 10. The normalized modal cyclic dispersion coefficient [the term between square brackets in Eq. (47)] as a function of the dimensionless reversal time $\left(\mathrm{t}_{r} / \tau_{n}\right)$.

$$
D_{\text {micro }}=D_{m o l}+\frac{1}{2} v_{n_{2}}^{2} \tau_{2},
$$

and only describe the relaxation of the larger scale. This reduces the velocity variance to the contribution by the scale undergoing relaxation,

$$
\sigma_{v, r e l}^{2}=\frac{1}{2} v_{n_{1}}^{2} \tau_{1}
$$

and changes the variance of the approximate model to

$$
\sigma_{a p, x, r e l}^{2}=2 D_{m i c r o} t+v_{n_{1}}^{2} \tau_{1}\left(\tau_{1}\left[e^{-t / \tau_{1}}-1\right]+t\right) .
$$

With this scale separation, we obtain an excellent fit with the exact variance (Fig. 13).

If we put a scale (say n) in a small-scale dispersion coefficient and neglect its relaxation, the (relative) error we make in the contribution of this scale to the exact variance (15) is bounded,

$$
\begin{aligned}
\text { Error }= & \left|\frac{v_{n}^{2} \tau_{n}\left(t+\left[e^{-t / \tau_{n}}-1\right]\right)-v_{n}^{2} \tau_{n} t}{v_{n}^{2} \tau_{n} t}\right| \leqslant\left|\frac{v_{n}^{2} \tau_{n}^{2}}{v_{n}^{2} \tau_{n} t}\right| \\
& =\frac{\tau_{n}}{t}=\frac{1}{t_{n, o b s}} .
\end{aligned}
$$

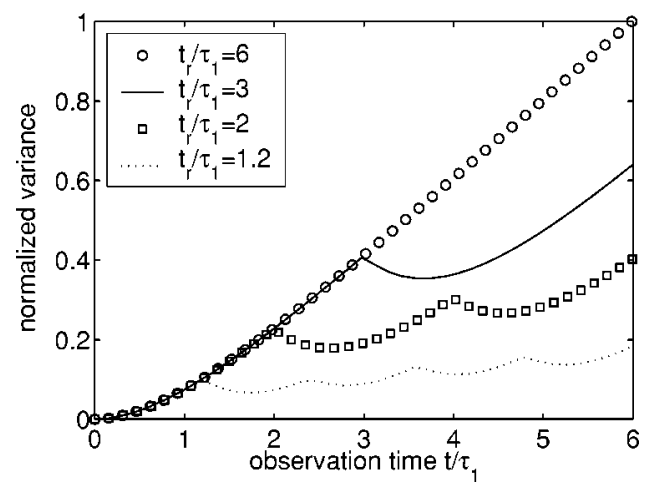

FIG. 11. Effect of the dimensionless reversal time $\left(t_{r} / \tau_{1}\right)$ on the development of the variance (normalized at the variance for unidirectional flow). 


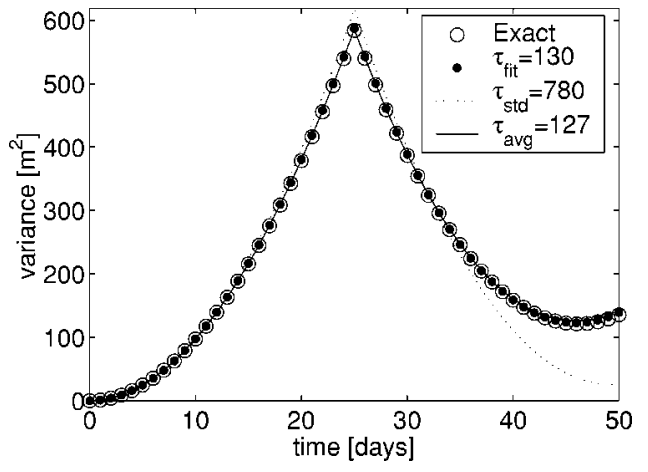

FIG. 12. Comparison of the exact variance with the variance by the fourth-other upscaled model for a bimodal velocity field (49) with $\mathrm{n}_{1}=1$ and $\mathrm{n}_{2}=5$. In the fourth-order model, the relaxation time is used as fit parameter $\left(\tau_{f i t}\right)$ or is defined as $\tau_{\text {std }}(28)$ and $\tau_{\text {avg }}(52)$, respectively.

This relative error decreases for increasing observation time. We now postulate a separation criterion based on this single scale analysis. Scale (n) is added to the microscale dispersion coefficient in the approximation and its relaxation ignored if the relative error bound (56) drops below a certain error value $\varepsilon$,

$$
\frac{\tau_{n}}{t}=\frac{1}{n^{2}} \frac{\tau_{1}}{t}=\frac{1}{n^{2}} \frac{1}{t_{o b s}} \leqslant \varepsilon \Leftrightarrow n \geqslant \sqrt{\varepsilon / t_{o b s}} .
$$

We define the observation time-dependent separation scale $n_{\text {sep }}\left(t_{o b s} ; \varepsilon\right)$ as the real-valued (n) for which the equality in Eq. (57) holds,

$$
n_{\text {sep }}\left(t_{\text {obs }} ; \varepsilon\right)=\sqrt{\varepsilon / t_{\text {obs }}}
$$
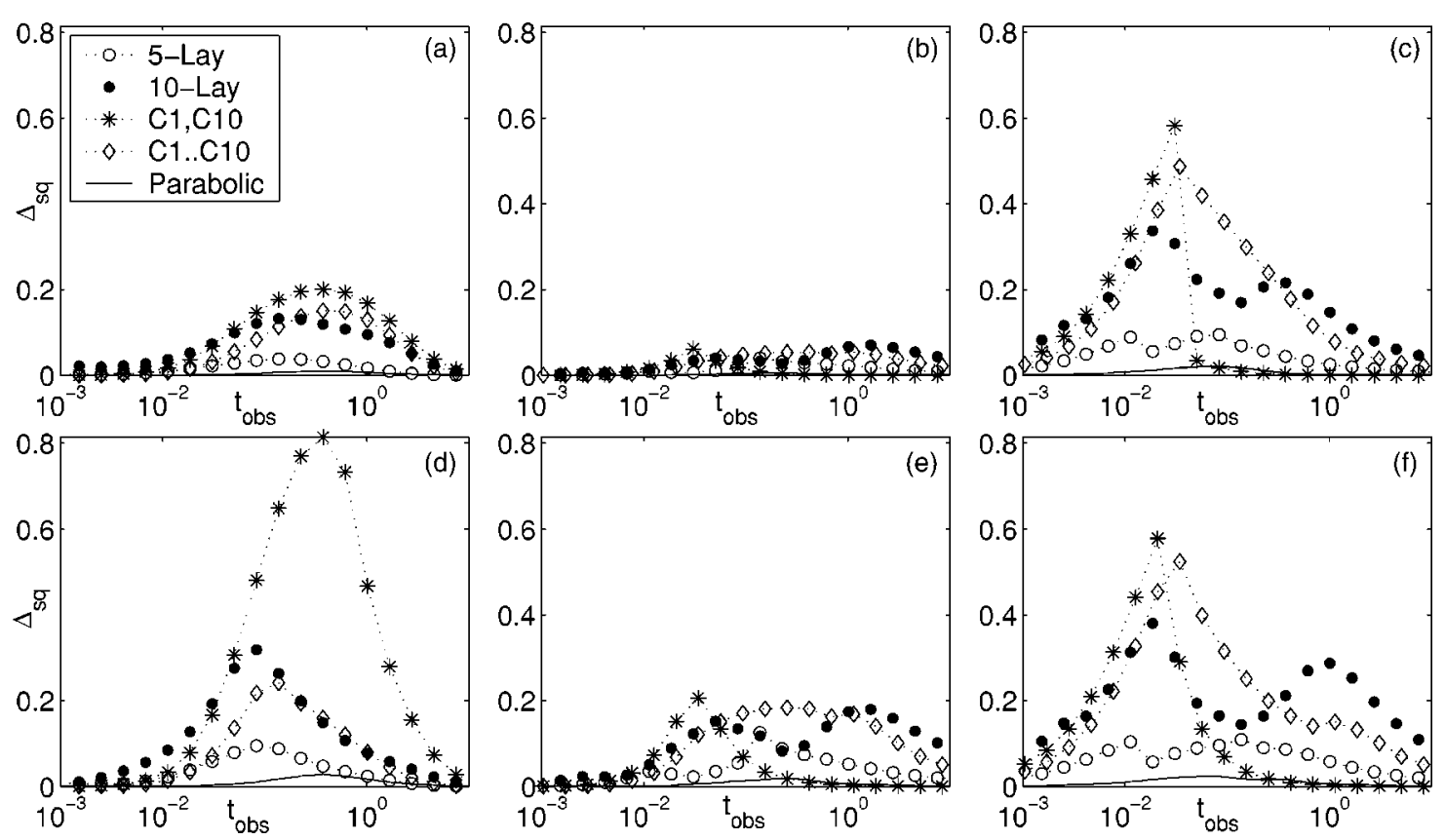

FIG. 14. Error $\Delta_{\mathrm{sq}}(66)$ for the velocity fields of Table I as a function of the observation time. (a,d) No scale separation; optimization of $\tau$ only. (b,e) Scale separation with $n_{\text {sep }}(58)$ and $\tau_{\text {avg }}(64)$. (c,f) Scale separation with $n_{\text {sep }}(58)$ and $\tau_{\text {std }}(28)$. The errors (a,b,c) are for unidirectional flow. The errors (d,e,f) for flow including reversal of direction. The $\alpha$ and $\epsilon$ values were found by trial and error. The results in (b) and (c) are obtained for $\epsilon=6.2$ and $\alpha=0.5$ 
Here the observation-time-dependent scale separator $\mathrm{n}_{\text {sep }}\left(\mathrm{t}_{\mathrm{obs}} ; \varepsilon\right)$ is defined according to Eq. (58) and the function $\mathrm{N}^{+}$is the first integer larger than $\mathrm{n}_{\text {sep }}\left[N^{+}(x)=\min _{n \in \mathbb{N}}\{n\right.$ $>x\}$ ]. Filter $\omega^{*}\left(t_{o b s} ; n\right)(59)$ is defined such that the contribution of each mode to the relaxation part smoothly vanishes in time, in descending mode order. At a specific point in time, only a single scale [mode $\left.\mathrm{n}=\mathrm{N}^{+}\left(\mathrm{n}_{\text {sep }}\right)\right]$ is partitioned over the relaxation part and $\mathrm{D}_{\text {micro }}$. In the limit of the observation time to infinity, the separator scale $n_{\text {sep }}$ (58) becomes zero and according to Eq. (59) all scales are described by an effective dispersion coefficient. However, for the accuracy of the approximation it is always favorable to at least describe the relaxation of a part of the largest scales. We relate this part to the (normalized) contribution of all modes to the effective dispersion coefficient in the Fickian limit. Let us define mode $\mathrm{n}_{\min }(\alpha)$ as

$$
n_{\min }(\alpha)=\min _{n \in \mathbb{N}}\left\{n \mid \sum_{k=1}^{n} v_{k}^{2} \tau_{k} \geqslant \alpha D_{e f f, \infty}\right\} .
$$

Given $\alpha$, all modes up to mode $\mathrm{n}_{\min }$ will be fully described by the relaxation part independent of the magnitude of the observation time. With this modification, the fraction of scale $\mathrm{n}\left[\omega\left(t_{o b s} ; n\right)\right]$ that is put in the relaxation part finally reads

$$
\omega\left(t_{o b s} ; n\right)=\left\{\begin{array}{ll}
1, & n \leqslant n_{\min }(\alpha) \\
\omega^{*}\left(t_{o b s} ; n\right), & n>n_{\min }(\alpha)
\end{array} .\right.
$$

In the upscaled approximation including scale separation, the microscale dispersion coefficient consists of the molecular diffusion and the fractions of the scales that are relaxed,

$$
D_{\text {micro }}=D_{\text {mol }}+\sum_{n=1}^{\infty}\left[1-\omega\left(t_{o b s} ; n\right)\right] v_{n}^{2} \tau_{n} .
$$

The relaxation part of the velocity variance $\left(\sigma_{v, r e l}^{2}\right)$ describes the fraction of those velocity modes which undergo relaxation,

$$
\sigma_{v, r e l}^{2}=\frac{1}{2} \sum_{n=1}^{\infty} \omega\left(t_{o b s} ; n\right) v_{n}^{2} .
$$

We compute the relaxation time of this velocity fraction analogously to Eq. (52),

$$
\tau_{\text {avg }}=\frac{1}{\Delta t} \int_{t_{\min }}^{t_{\max }} \frac{\sum_{n=1}^{\infty} \omega\left(t_{o b s} ; n\right) v_{n}^{2} \tau_{n}\left(1-e^{-t / \tau_{n}}\right)}{\sum_{n=1}^{\infty} \omega\left(t_{o b s} ; n\right) v_{n}^{2}\left(1-e^{-t / \tau_{n}}\right)} d t .
$$

With these definitions, the microscale dispersion coefficient $\mathrm{D}_{\text {micro }}(62)$ replaces the molecular diffusion coefficient $\mathrm{D}_{\text {mol }}$ in Eq. (2). In Eq. (22), the relaxation part of the variance $\sigma_{v, r e l}^{2}(63)$ is substituted for the total velocity variance $\sigma_{v}^{2}$ and $\tau_{\text {avg }}$ (64) replaces the relaxation time $\tau$. The corresponding time derivative of the variance reads

$$
\partial_{t} \sigma_{a p, x, \text { rel }}^{2}(t)=2 \times \begin{cases}D_{\text {micro }}+\sigma_{v, \text { rel }}^{2} \tau_{\text {avg }}\left(1-e^{\left.-t / \tau_{\text {avg }}\right),}\right. & t \leqslant t_{r} \\ D_{\text {micro }}+\sigma_{v, \text { rel }}^{2} \tau_{\text {avg }}\left(1-\left[2 e^{+t, / \tau_{\text {avg }}}-1\right] e^{\left.-t / \tau_{\text {avg }}\right),}\right. & t>t_{r} .\end{cases}
$$

\section{E. Optimization and evaluation}

The upscaled model with scale separation may be interpreted as a function of two independent variables, the separation scale $(\mathrm{N})$ and the relaxation time $(\tau)$. We want to quantify the accuracy of the model with respect to the behavior of the variance in time. The error measure $\Delta_{s q}\left(\mathrm{n}_{\text {sep }}, \tau\right)$ computes the relative error in the time derivative of the variance that is made by the approximation (65) on a time domain $\left[\mathrm{t}_{\min }, \mathrm{t}_{\text {max }}\right]$ as

$$
\Delta_{s q}(N, \tau)=\frac{\int_{t_{\min }}^{t_{\max }}\left[\partial_{t} \sigma_{c, x}^{2}(t)-\partial_{t} \sigma_{a p, x}^{2}(t ; N, \tau)\right]^{2} d t}{\int_{t_{\min }}^{t_{\max }}\left[\partial_{t} \sigma_{c, x}^{2}(t)-2 D_{m o l}\right]^{2} d t} .
$$

Here $\partial_{t} \sigma_{a p, x}^{2}$ is the time derivative of the variance by the approximation (65) and $\partial_{t} \sigma_{c, x}^{2}$ the exact variance time derivative (15).

\section{F. Test fields}

We test the upscaled model for a parabolic velocity field, two cosine velocity fields, and two layered velocity fields. The latter two consist of equally sized layers of different velocities, as shown in Table I. We consider flow with and without flow reversal. For each field, we vary the observation time $\mathrm{t}_{o b s}$ in the range $t_{o b s} \in\left[10^{-3}, 10^{1}\right]$. For each $\mathrm{t}_{o b s}$, we compute $\left(\mathrm{n}_{\text {sep }}, \tau_{\text {avg }}\right)\left[\right.$ [Eqs. (58) and (64)] and $\left(\mathrm{n}_{\text {sep }}, \tau_{\text {std }}\right)$ [Eqs. (58) and (28)]. We evaluate the approximations as function of $\mathrm{t}_{o b s}$ by comparing the error $\left(\Delta_{s q}\right)$ evaluated on the time range $\left[t_{\min }, t_{\max }\right]=[0,2] \tau_{1} t_{o b s}$.

\section{G. Results}

Figures 14(b) and 14(e) show that the scale separator $\mathrm{n}_{\text {sep }}$ (58) combined with relaxation time $\tau_{a v g}$ (64) gives small errors. Moreover, the results are almost indistinguishable from those obtained by independently optimizing $\mathrm{n}$ and $\tau$ with respect to $\Delta_{s q}(N, \tau)$. On the contrary, the same scale separator combined with the standard relaxation time $\tau_{\text {std }}$ (28) gives errors that are much larger [Figs. 14(c) and 14(f)]. 
TABLE I. Description of the velocity fields $\mathrm{v}(\mathrm{y})$ under consideration ( $\mathrm{d}$ is the height of the field) (in $10^{-1} \mathrm{~m} / \mathrm{d}$ ).

\begin{tabular}{ccccc}
\hline \hline Parabolic & $\cos 1+\cos 10$ & $\sum \cos (1 \cdots 10)$ & 5 layer & 10 layer \\
\hline $15\left[1-(2 y / d-1)^{2}\right]$ & $\cos (\pi y / d)$ & 10 & layer velocities & layer velocities \\
& $+\cos (10 \pi y / d)$ & $\sum_{n=1} \cos (n \pi / d y)$ & $\mathrm{v}=[1,3,9,1,8]$ & $\mathrm{v}=[3,2,12,7,13,1,8,5,4,13]$ \\
\hline \hline
\end{tabular}

As mentioned, Eq. (28) overestimates the relaxation time by overemphasizing the relaxation times of large scales for short times. In case we do not apply scale separation, the errors are large [Figs. 14(a) and 14(d)]. For unidirectional flow, the relaxation time has a bigger impact than scale separation. For flow including reversal, the largest errors are made if we do not separate scales [Fig. 14(d)].

\section{DISCUSSION}

Hassanizadeh [19] and Tompson and Gray [20] each derived a macroscopic multidimensional non-Fickian dispersion model. Hassanizadeh [19] derived his equation by exploring the mass and momentum balances for a solute and solvent at the macroscale. Tompson and Gray [20] applied the method of volume averaging on the convection diffusion equation. Although the model of Camacho [Eqs. (8) and (39)] is derived from upscaling to the megascopic scale to a $1 \mathrm{D}$ representation, it has the same functional form. The equivalence of these models suggests that dispersion at the macroscale might be interpreted as multidimensional Taylor dispersion. In Verlaan [21] and Berentsen [18] it is shown that the fourth-order model is able to explain the scale dependency of the dispersivity observed in echo experiments measured by Rigord et al. [10]. Moreover, they showed that in a qualitative sense the fourth-order model is able to reproduce the dependency of the effective dispersion coefficient on the Péclet number (see Bear [22]).

\section{CONCLUSIONS}

The fourth-order approximation as proposed by Camacho provides acceptable results only in the case of single scale problems and uniform initial conditions. We characterized the scale interaction by analyzing the moments derived through spectral analysis. The analysis allowed us to formulate an upscaled model with effective parameters that can be calculated a priori from the velocity distribution. Separation of scales in combination with a new definition of the effective relaxation time allowed us to extend the model to obtain good results also for multiscale problems. In addition, we studied the effects of periodic flow reversal. Periodic flow reversal results in relaxation of the moments to periodic behavior in the same relaxation time as for the unidirectional flow, even if the reversal time is smaller than the relaxation time. The effective dispersion coefficient is a monotonously increasing function of the dimensionless cycle time $\left(\mathrm{t}_{r} / \tau\right)$. It asymptotically converges for increasing $\mathrm{t}_{r} / \tau$ towards the effective dispersion coefficient in the absence of any flow reversal.

\section{APPENDIX A: EXACT SPATIAL MOMENTS OF THE 2D CONVECTION DIFFUSION EQUATION}

\section{Mean position of higher concentration modes}

\section{Unidirectional flow}

The evolution expression of the non-normalized first moment of mode $n$, Eq. (9), for $\mathrm{k}=1$, reads

$$
\begin{aligned}
& {\left[\tau_{n}^{-1}+\frac{\partial}{\partial t}\right] E_{c_{n}, x, 1}=\frac{1}{2}\left[2 v_{0}+v_{2 n}\right] M_{c_{n}},} \\
& +v_{n} M_{0}+\frac{1}{2} \sum_{m=1 \neq n}^{\infty}\left[v_{m+n}+v_{|m-n|}\right] M_{c_{m}} .
\end{aligned}
$$

We replace $\mathrm{M}_{c_{n}}(\mathrm{t})$ and $\mathrm{M}_{c_{m}}(\mathrm{t})$ with Eq. (11) on the RHS of Eq. (A1) and solve the result for the initial condition, $E_{c_{n}, x, 1}(0)=x_{0} M_{c_{n}}^{\text {init }}$, since all tracer is released at $\mathrm{x}=\mathrm{x}_{0}$. For $E_{c_{n}, x, 1}(\mathrm{t})$, one obtains

$$
\begin{aligned}
E_{c_{n}, x, 1}(t)= & M_{c_{n}}^{i n i t} x_{0} e^{-t / \tau_{n}}+v_{n} M_{0} \tau_{n}\left[1-e^{-t / \tau_{n}}\right] \\
& +\sum_{m=1 \neq n}^{\infty} \frac{\tau_{n} \tau_{m}}{\tau_{m}-\tau_{n}} \frac{v_{m+n}+v_{|m-n|}}{2} M_{c_{m}}^{i n i t}\left[e^{-t / \tau_{m}}-e^{-t / \tau_{n}}\right] \\
& +\left[v_{0}+\frac{v_{2 n}}{2}\right] M_{c_{n}}^{i n i t} t e^{-t / \tau_{n}}
\end{aligned}
$$

Flow reversal

The evolution expression of $E_{c_{n}, x, 1}^{r e v}(t)$ for flow after reversal is equal to Eq. (A1) with the signs of the modal velocities changed,

$$
\begin{aligned}
{\left[\tau_{n}^{-1}+\frac{\partial}{\partial t}\right] E_{c_{n}, x, 1}^{r e v}=} & -\frac{1}{2}\left[2 v_{0}+v_{2 n}\right] M_{c_{n}}(t)-v_{n} M_{0} \\
& -\frac{1}{2} \sum_{m=1 \neq n}^{\infty}\left[v_{m+n}+v_{|m-n|}\right] M_{c_{m}}(t) .
\end{aligned}
$$

The solution to Eq. (A3) is found by replacing $\mathrm{M}_{c_{n}}(\mathrm{t})$ and $\mathbf{M}_{c_{m}}$ with Eq. (11) and using continuity of $\mathrm{E}_{c_{n}, x, 1}$ at the moment of reversal, $E_{c_{n}, x, 1}^{r e v}\left(t_{r}\right)=E_{c_{n}, x, 1}\left(t_{r}\right)$, and yields

$$
\begin{aligned}
E_{c_{n}, x, 1}^{r e v}(t)= & -\left[v_{0}+\frac{v_{2 n}}{2}\right] M_{c_{n}}^{i n i t}\left(t-2 t_{r}\right) e^{-t / \tau_{n}}+M_{c_{n}}^{i n i t} x_{0} e^{-t / \tau_{n}} \\
& -v_{n} M_{0} \tau_{n}\left[1-2 e^{-\left(t-t_{r}\right) / \tau_{n}}+e^{-t / \tau_{n}}\right] \\
& -\sum_{m=1 \neq n}^{\infty} \frac{\tau_{n} \tau_{m}}{\tau_{m}-\tau_{n}} \frac{v_{m+n}+v_{|m-n|}}{2} M_{c_{m}}^{i n i t} \times\left(e^{-t / \tau_{m}}\right. \\
& \left.-\left[2 e^{\left[\left(\tau_{m}-\tau_{n}\right) / \tau_{m} \tau_{n}\right] t_{r}}-1\right] e^{-t / \tau_{n}}\right)
\end{aligned}
$$




\section{Variance of average concentration}

\section{Unidirectional flow}

The evolution equation for the second moment belonging to $\mathrm{c}_{0}(\mathrm{x}, \mathrm{t})$ reads [Eq. (8) for $\mathrm{k}=2$ ]

$$
\frac{\partial M_{c_{0}, x, 2}}{\partial t}=2 D_{m o l}+2 v_{0} \mu_{c_{0}, x}+\sum_{n=1}^{\infty} \frac{v_{n}}{M_{0}} E_{c_{n}, x, 1} .
$$

Using, $\sigma_{c, x}^{2}=M_{c_{0}, x, 2}-\mu_{c_{0}, x}^{2}$, the evolution equation for the centered second (variance) yields

$$
\frac{\partial \sigma_{c, x}^{2}}{\partial t}=2 D_{m o l}+\left[2 v_{0}-2 \frac{\partial \mu_{c_{0}, x}}{\partial t}\right] \mu_{c_{0}, x}(t)+\sum_{n=1}^{\infty} \frac{v_{n}}{M_{0}} E_{c_{n}, x, 1}(t) .
$$

We substitute the known expressions for $\mu_{c_{0}, x}$ (13) and $E_{c_{n}, x, 1}(\mathrm{~A} 2)$ on the right-hand side and find expression (15) as the solution to the initial condition $\sigma_{c, x}^{2}(0)=0$.

\section{Flow reversal}

The evolution expression for the variance for flow after reversal is equivalent to Eq. (A6) with the signs of the velocity changed,

$$
\begin{aligned}
\frac{\partial \sigma_{c, x, r e v}^{2}}{\partial t}= & 2 D_{m o l}+\left[-2 v_{0}-2 \frac{\partial \mu_{c_{0}, x}^{r e v}}{\partial t}\right] \mu_{c_{0}, x}^{r e v}(t) \\
& -\sum_{n=1}^{\infty} \frac{v_{n}}{M_{0}} E_{c_{n}, x, 1}^{r e v}(t) .
\end{aligned}
$$

The solution (39) to this ODE is obtained by substituting Eq. (36) for $\mu_{c, x}^{r e v}$ and Eq. (A4) for $E_{c_{n}, x, 1}^{r e v}$ and using the continuity of the variance at the moment of reversal, $\sigma_{c, x, r e v}^{2}\left(t_{r}\right)$ $=\sigma_{c, x}^{2}\left(t_{r}\right)$. For a more detailed derivation, see [18].

\section{APPENDIX B: SPATIAL MOMENTS OF THE FOURTH- ORDER UPSCALED EQUATION}

Multiplication of the fourth-order equation (23) with $\mathrm{x}^{k}$, integrating the result over the $\mathrm{x}$ domain, gives the following general evolution equation for the $k$ th moment of the concentration:

$$
\begin{aligned}
\tau \frac{\partial^{2} M_{c, x, k}^{a p}}{\partial t^{2}}+\frac{\partial M_{c, x, k}^{a p}}{\partial t}= & k v_{0} M_{c, x, k-1}^{a p}+k\left(2 v_{0}+\beta\right) \frac{\partial M_{c, x, k-1}^{a p}}{\partial t}+\left(k^{2}\right. \\
& -k)\left[D_{m o l}+\sigma_{v}^{2} \tau-\left(v_{0}+\beta\right) v_{0} \tau\right] M_{c, x, k-1}^{a p} \\
& +2\left(k^{2}-k\right) D_{m o l} \tau \frac{\partial M_{c, x, k-2}^{a p}}{\partial t} \\
& -\left(\prod_{i=0}^{2}(k-i)\right) D_{m o l}\left(\beta+2 v_{0}\right) \tau M_{c, x, k-3}^{a p} \\
& -\left(\prod_{i=0}^{3}(k-i)\right) D_{m o l}^{2} \tau M_{c, x, k-4}^{a p} .
\end{aligned}
$$

The moments of the Telegraph equation are obtained by taking $\mathrm{D}_{m o l}$ equal to zero in Eq. (B1).

\section{Mean particle position}

The evolution expression for the mean position of the average concentration yields

$$
\tau \frac{\partial^{2} \mu_{c, x}^{a p}}{\partial t^{2}}+\frac{\partial \mu_{c, x}^{a p}}{\partial t}=-v_{0}
$$

Initially all particles are released at $\mathrm{x}_{0}$. If the particles are initially distributed nonuniformly over the height, the initial particle velocity of the particles is $\mathrm{u}_{0}$, which may be different from $v_{0}$,

$$
\mu_{c, x}^{a p}(0)=x_{0}, \frac{\partial \mu_{c, x}^{a p}}{\partial t}(0)=u_{0}
$$

The solution of Eq. (B2) subject to result is given by Eq. (24).

\section{Spatial variance in the particle distribution}

The evolution expression for the second normalized moment reads

$$
\begin{aligned}
\tau \frac{\partial^{2} M_{c, x, 2}^{a p}}{\partial t^{2}}+\frac{\partial M_{c, x, 2}^{a p}}{\partial t}= & 2\left(2 v_{0}+\beta\right) \tau \frac{\partial \mu_{c, x}^{a p}}{\partial t} \\
& +2 v_{0} \mu_{c, x}^{a p}+2\left[D_{m o l}+\sigma_{v}^{2} \tau-\left(v_{0}+\beta\right) v_{0}\right]
\end{aligned}
$$

Using $\sigma_{c, x}^{a p, 2}=M_{c, x, 2}^{a p}-\left(\mu_{c, x}^{a p}\right)^{2}$ and the expressions for $\mu_{c, x}^{a p}$ given in Eq. (24), the following evolution equation for the variance is obtained:

$$
\begin{aligned}
\tau \frac{\partial^{2} \sigma_{c, x}^{a p, 2}}{\partial t^{2}}+\frac{\partial \sigma_{c, x}^{a p, 2}}{\partial t}= & 2\left(D_{m o l}+\sigma_{v}^{2} \tau\right)+2 \tau \beta\left(u_{0}-v_{0}\right) e^{-t / \tau} \\
& -2 \tau\left(u_{0}-v_{0}\right)^{2} e^{-2 t / \tau}
\end{aligned}
$$

Initially, the variance is zero while the initial increase of the variance is by molecular diffusion only,

$$
\sigma_{c, x}^{a p, 2}(0)=0, \quad \frac{\partial \sigma_{c, x}^{a p, 2}}{\partial t}(0)=2 D_{m o l} .
$$

The solution of Eq. (B5) with respect to Eq. (B6) is given by Eq. (26).

\section{APPENDIX C: PERIODIC FLOW REVERSAL}

The differential equation (in a moving frame of reference) for the first moment of a higher-order concentration mode in the $\left(\mathrm{N}_{r}\right)$ th reversal cycle reads

$$
\left[\frac{\partial}{\partial t}+\frac{1}{\tau_{n}}\right] E_{c_{n}, x, 1}(t)=(-1)^{N_{r}} v_{n} M_{0} .
$$

The first moment $\left(E_{c_{n}, x, 1}\right)$ is initially zero and continuous each time the flow is reversed. Consequently, the solution to Eq. (C1) is given by

$$
E_{c_{n}, x, 1}(t)=-2 M_{0} v_{n} \tau_{n} \sum_{k=0}^{N_{r}}{ }^{\prime}(-1)^{k}\left(e^{-\left(t-k t_{r}\right) / \tau_{n}}-1\right) .
$$


The time derivative of the variance is, similar to Eq. (A5), directly related to the first moment of the higher-order concentration modes and reads

$$
\frac{\partial \sigma_{c, x}^{2}}{\partial t}(t)=2 D_{m o l}-\sum_{n=1}^{\infty}(-1)^{N_{r}} v_{n} \frac{E_{c_{n}, x, 1}}{M_{0}}(t) .
$$

Substituting Eq. (C2) into Eq. (C3) yields

$$
\begin{aligned}
\frac{\partial \sigma_{c, x}^{2}}{\partial t}\left(\tilde{t} ; N_{r}, t_{r}\right)= & 2 D_{m o l}-2 \sum_{n=1}^{\infty} v_{n}^{2} \tau_{n}\left[\sum_{k=0}^{N_{r}}(-1)^{N_{r}+k}\right. \\
& \left.\times\left[e^{-\left[\tilde{t}+\left(N_{r}-k\right) t_{r}\right] \tau_{n}}-1\right]\right] .
\end{aligned}
$$

Evaluation of the sum gives expression (45) in Sec. IX A.
[1] L. W.Lake and G. J.Hirasaki, SPEJAugust, 459 (1981).

[2] L.W. Gelhar, Stochastic Subsurface Hydrology (Prentice-Hall, Englewood Cliffs, NJ, 1993).

[3] G.I. Taylor, Proc. R. Soc. London, Ser. A 219, 186 (1953).

[4] G. I.Taylor, Proc. R. Soc. London, Ser. A 225, 473 (1954).

[5] R. Aris, Proc. R. Soc. London, Ser. A 235, 67 (1956).

[6] P. G.Saffman, J. Fluid Mech. 6, 321 (1959).

[7] P.G. Saffman, J. Fluid Mech. 7, 194 (1960).

[8] G. De Josselin de Jong, Trans., Am. Geophys. Union 39, 67 (1958).

[9] J. W.Hiby, in Symposium on the Interaction Between Fluids and Particles, edited byP.A.Rottenburg and N.T.Shepherd(The Institution of Chemical Engineeers, London, 1962), p. 312.

[10] P. Rigord, A. Calvo, and J. P.Hulin, Phys. Fluids A 2, 681 (1990).

[11] M. W.Legatski and D.L. Katz, SPE J. March, 43 (1967).

[12] J. H.Cushman, The Physics of Fluids in Hierarchical Porous Media: Angstroms to Miles (Kluwer, Dordrecht, 1997).

[13] D.L. Strack, Water Resour. Res. 28, 2973 (1992).

[14] A. E.Scheidegger, Can. J. Phys. 36, 649 (1958).

[15] J. Camacho, Phys. Rev. E 47, 1049 (1993).

[16] J. Camacho, Phys. Rev. E 48, 310 (1993).

[17] G. Marinoschi and H. Vereecken, Transp. Porous Media 45, 367 (2001).

[18] C. W.J.Berentsen, Ph.D. thesis, Delft University of Technology, Delft (2003).

[19] S. M.Hassanizadeh, Transp. Porous Media 23, 107 (1996).

[20] A.F.B. Tompson and W.G. Gray, Water Resour. Res. 22, 591 (1986).

[21] M. L.Verlaan, Ph.D. thesis, Delft University of Technology,
Delft (2001).

[22] J. Bear, Dynamics of Fluid in Porous Media (Dover Publications, New York, 1972).

[23] M. L.Verlaan, C.W.J. Berentsen, C.P.J.W. van Kruijsdijk, and E. F. Nederlof, 7th European Conference on the Mathematics of Oil Recovery, Baveno, Italy (EAGE, Houten, 2000).

[24] The tracer concentration does not affect the fluid flow behavior. The fluid velocity and the fluid density are independent of the tracer concentration.

[25] In Verlaan et al. [21,23] and Berentsen [18], microscopic dispersion is included in the small-scale dispersion mechanism.

[26] More precisely, the relaxation time of a single scale is the characteristic time that expresses the exponential decay of that scale $[\exp (-t / \tau)]$. The relaxation time of a multiscale problem is the time scale that characterizes the combined decay of all scales present in the problem.

[27] We use $\beta$ in stead of $\beta \mathrm{v}_{0}$ used by Camacho, since the modal velocity interactions may be nonzero $\left(\gamma_{m n} \neq 0\right)$ if the mean velocity is zero $\left(\mathrm{v}_{0}=0\right)$.

[28] Taylor dispersion is a process that has a finite propagation speed. An important disadvantage of using the uCDifE is that it results in an infinite propagation speed. The Telegraph model recovers the finite propagation speed by ignoring longitudinal diffusion which is a secondary process compared to Taylor dispersion.

[29] The superscript/subscript (rev) refers to the situation after reversal of the flow direction.

[30] As microscale dispersion has been eliminated to obtain the Telegraph model, scale separation cannot be applied to this model. 